LIMNOLOGICAL SURVEY OF SACONY CREEK BASIN,

BERKS COUNTY, PENNSYLVANIA

U.S. GEOLOGICAL SURVEY

Water Resources Investigations

$76-84$

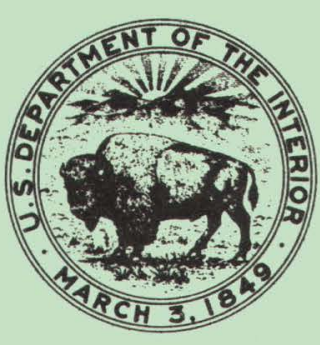

Prepared in cooperation with

U.S. Department of Agriculture,

Soil Conservation Service 


\begin{tabular}{|c|c|}
\hline $\begin{array}{l}\text { BIBLIOGRAPHIC DATA } \\
\text { SHEET }\end{array}$ & 3. Recipient's Accession No. \\
\hline \multirow{2}{*}{$\begin{array}{l}\text { 4. Title and Subtitle } \\
\text { Limnological Survey of Sacony Creek Basin, Berks County } \\
\text { Pennsylvania }\end{array}$} & \begin{tabular}{|l|} 
5. Report Date \\
August 1976 \\
\end{tabular} \\
\hline & 6. \\
\hline $\begin{array}{l}\text { 7. Author(s) } \\
\text { James L. Barker and Kenneth P. Kulp }\end{array}$ & $\begin{array}{l}\text { 8. Performing Organization Rept. } \\
\text { No. USGS/WRI 76-84 } \\
\end{array}$ \\
\hline $\begin{array}{l}\text { 9. Performing Organization Name and Address } \\
\text { U.S. Geological Survey, Water Resources Division } \\
228 \text { Walnut Street, P. O. Box } 1107 \\
\text { Harrisburg, Pennsylvania } 17108\end{array}$ & 10. Project/Task/Work Unit No. \\
\hline \multirow{2}{*}{$\begin{array}{l}\text { 12. Sponsoring Organization Name and Address } \\
\text { U.S. Geological Survey, Water Resources Division } \\
228 \text { Walnut Street, P. O. Box } 1107 \\
\text { Harrisburg, Pennsylvania } 17108\end{array}$} & $\begin{array}{l}\text { 13. Type of Report \& Period } \\
\text { Covered } \\
\text { Final } \\
\end{array}$ \\
\hline & 14. \\
\hline \multicolumn{2}{|c|}{$\begin{array}{l}\text { 15. Supplementary Notes Prepared in cooperation with the U.S. Department of Agriculture, } \\
\text { Soil Conservation Service. }\end{array}$} \\
\hline \multicolumn{2}{|c|}{$\begin{array}{l}\text { 16. Abstracts Samples of water, fish, and benthic macroinvertebrates collected at } 10 \\
\text { sampling stations over a } 10 \text {-month period indicate that Sacony Creek and its major } \\
\text { tributaries possess water of good to excellent quality. No excessive quantities of } \\
\text { dissolved nutrients, oxidizable matter, or fecal coliform bacteria were detected. } \\
\text { Fish inhabitants include a sizable wild trout population in the upper Sacony basin and } \\
\text { a diverse warm-water population, dominated by white suckers (Catostomus commersoni), } \\
\text { in the lower basin. A population of } 590 \text { trout or } 119 \text { pounds per acre (133 kilograms } \\
\text { per square hectometre) was estimated for good habitat in the upper basin. A maximum } \\
\text { standing crop of } 558 \text { pounds per acre ( } 626 \text { kilograms per square hectometre) was } \\
\text { measured at one of the more productive reaches. Benthic macroinvertebrate collections } \\
\text { tended to support the water chemistry and fish population studies. Diversity (d) and } \\
\text { Redundancy ( } r \text { ) ranged from } 2.44 \text { to } 3.46 \text { and } 0.14 \text { to } 0.38 \text {, respectively. Such } \\
\text { diversity and redundancy values indicate good quality water at all stations. }\end{array}$} \\
\hline
\end{tabular}

17. Key Words and Document Analysis. 17a. Descriptors

Aquatic environment, benthic forms, limnology, watershed, habitat, fish population, trout, biomass

17b. Identifiers/Open-Ended Terms

Limnology survey, Delaware River basin

17c. COSATI Field/Group

18. Availability Statement

No Restriction on Distribution

\begin{tabular}{|l|l|}
\begin{tabular}{|l|l|} 
19. Security Class (This \\
Report) \\
UNCLASSIFIED
\end{tabular} & $\begin{array}{c}\text { 21. No. of Pages } \\
45\end{array}$ \\
\hline $\begin{array}{l}\text { 20. Security Class (This } \\
\text { Page } \\
\text { UNCLASSIFIED }\end{array}$ & 22. Price \\
\hline RRM MAY BE REPRODUCED & USCOMM-DC 8265 -P74
\end{tabular}


LIMNOLOGICAL SURVEY OF SACONY CREEK BASIN,

BERKS COUNTY, PENNSYLVANIA

By James L. Barker and Kenneth P. Kulp

U.S. GEOLOGICAL SURVEY

Water Resources Investigations

$76-84$

Prepared in cooperation with

U.S. Department of Agriculture,

Soil Conservation Service

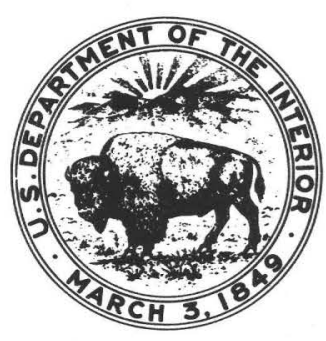

August 1976 
UNITED STATES DEPARTMENT OF THE INTERIOR

Thomas S. Kleppe, Secretary

GEOLOGICAL SURVEY

V. E. McKelvey, Director

For additional information write to:

U.S. Geological Survey

Fourth Floor, Federal Building

P. 0. Box 1107

Harrisburg, Pennsylvania 17108 
Factors for converting English units to International System of units (SI) -

Abstract --

Introduction --

Purpose and scope -_-

Description of study area -

Streamflow - - 10

Geology -- 4

Climatology -

Data collection - 4

Physio-chemical -----_-_-_-- 4

Biological -- 4

Fecal coliform bacteria - 4

Benthic macroinvertebrates --_---_--- 5

Fishes - 5

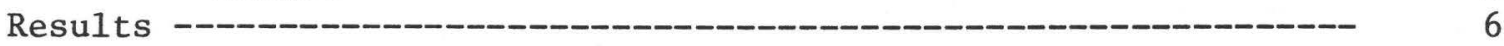

Physio-chemical -- 6

Biological -- 10

Fecal coliform bacteria - 10

Benthic macroinvertebrates --_-_-_---_--_---- 10

Fishes -- 10

Age composition of trout populations ----- 20

Estimates of trout populations --_-_-_-_-_-- 24

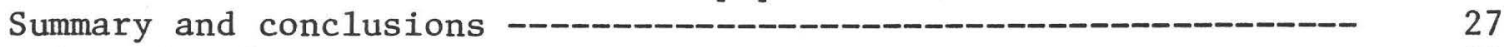

Selected references --

\section{ILLUSTRATIONS}

Figure 1. Map showing study area and sampling stations -------- 3

2. Diel fluctuation in dissolved oxygen and temperature-- 8

3. Graph showing species diversity and standing crop of fishes -- 21

4. Graph showing comparative length-age of Sacony Creek and Benner Springs hatchery brown trout --------- 
TABLES

Page

Table 1. Median or mean concentrations of chemical and bacteriological constituents in Sacony Creek

2. Rasin, July 1974 to May 1975 dissolved oxygen study in Sacony Creek

3. Population composition of benthic macroinvertebrates in Sacony Creek, September 24-27, 1974

4. Diversity (d) and redundancy (r) of Sacony Creek benthos, September 24-27, 1974

5. Numbers and standing crop of fish inhabiting selected reaches of Sacony Creek basin ------------

6. Length-age frequency of Sacony Creek brook trout --------

7. Length-age frequency of Sacony Creek brown trout

8. Population estimate of trout in selected reaches of Sacony Creek

9. Water-quality determinations in Sacony

Creek basin

FACTORS FOR CONVERTING ENGLISH UNITS TO INTERNATIONAL SYSTEM OF UNITS (SI)

\section{Multiply English units}

inches (in)

feet $(\mathrm{ft})$

yards (yd)

miles (mi)

acres

square miles ( $\left.\mathrm{mi}^{2}\right)$

cubic feet per second $\left(\mathrm{ft}^{3} / \mathrm{s}\right)$

pounds (1b)

pounds per acre (lb/acre)
By

25.4

.3048

.9144

1.609

4047

.4047

.004047

2.590

.02832

.454

1.1208
To obtain SI units

millimetres (mm)

metres (m)

metres (m)

kilometres $(\mathrm{km})$

square metres $\left(\mathrm{m}^{2}\right)$

square hectometres $\left(\mathrm{hm}^{2}\right)$

square kilometres $\left(\mathrm{km}^{2}\right)$

square kilometres $\left(\mathrm{km}^{2}\right)$

cubic metres per second $\left(\mathrm{m}^{3} / \mathrm{s}\right)$

kilograms (kg)

kilograms per square hectometre $\left(\mathrm{kg} / \mathrm{hm}^{2}\right)$ 


\title{
LIMNOLOGICAL SURVEY OF SACONY CREEK BASIN, \\ BERKS COUNTY, PENNSYLVANIA
}

By James L. Barker and Kenneth P. Kulp

\begin{abstract}
Samples of water, fish, and benthic macroinvertebrates collected at 10 sampling stations over a 10-month period indicate that Sacony Creek and its major tributaries contain water of good to excellent quality. The waters were found to be free of excessive quantities of dissolved nutrients, oxidizable matter, and fecal coliform bacteria.

Fish inhabitants include a sizable wild trout population in the upper Sacony basin and a diverse warm-water population, dominated by white suckers (Catostomus commersoni), in the lower basin. A population of 590 trout or 119 pounds per acre (133 kilograms per square hectometre) was estimated for good habitat in the upper basin. A maximum standing crop of 558.7 pounds per acre (626 kilograms per square hectometre) was measured at one of the more productive reaches.

Benthic macroinvertebrate collections tended to support the water chemistry and fish population studies. Diversity (d) and redundancy ( $r$ ) ranged from 2.44 to 3.46 and 0.14 to 0.38 , respectively. Such diversity and redundancy values indicate a healthy environment at all stations.
\end{abstract}




\section{INTRODUCTION}

\section{Purpose and Scope}

A limnological survey of Sacony Creek basin was conducted to establish a base of physical, chemical, and biological data pertaining to the general health of the stream. A secondary objective of the survey was to delineate that part of the creek supporting a resident trout population.

This investigation included the study of the aquatic ecosystem at 10 preselected stations within 7 reaches of Sacony Creek. Determined were the general water chemistry, fecal coliform bacteria, the population and biomass of fishes, and diversity of benthic macroinvertebrates.

The study area included Sacony Creek and its major tributaries from its headwaters to the Greenwich Bridge north of Kutztown, a distance of about $7.5 \mathrm{mi}(12.07 \mathrm{~km})$.

The fishes and macroinvertebrates were collected for this investigation between September 24, 1974, and October 2, 1974, except for fishes at Sacony Creek tributary near Bowers (Station 4) which were collected on May 14, 1975. Water chemistry and bacteria analyses were conducted bimonthly from July 1974 to May 1975.

Description of the Study Area

Sacony Creek has its headwaters in the South Mountain and Reading Prong regions of southeastern Berks County. The Sacony flows north and west for approximately $13 \mathrm{mi}(20.9 \mathrm{~km}$ ) before joining Maiden Creek at Virginville. The study area map (fig. 1) shows the Sacony Creek watershed above Greenwich Bridge, the farthest downstream sampling site. The drainage area of the Sacony is $55.3 \mathrm{mi}^{2}\left(143 \mathrm{~km}^{2}\right)$.

\section{Streamf low}

The average flow of. Sacony Creek at its mouth is about $100 \mathrm{ft}^{3} / \mathrm{s}$ $\left(2.8 \mathrm{~m}^{3} / \mathrm{s}\right)$, based on the flow of the Schuylkill River at Berne. The minimum discharge measured was $1.33 \mathrm{ft}^{3} / \mathrm{s}\left(0.04 \mathrm{~m}^{3} / \mathrm{s}\right)$ on September 3, 1963. While no flood discharges have been measured, a maximum of about 4,000 $\mathrm{ft}^{3} / \mathrm{s}(113 \mathrm{~m} / \mathrm{s})$ was probable during the June 1972 tropical storm "Agnes." Serious flooding in the borough of Kutztown resulted from a discharge of that magnitude.

Streamflow conditions during the survey were near normal for the period. A discharge of $12.8 \mathrm{ft}^{3} / \mathrm{s}(0.36 \mathrm{~m} / \mathrm{s})$ was measured at the Greenwich Bridge (Station 10) on September 24, 1974. 


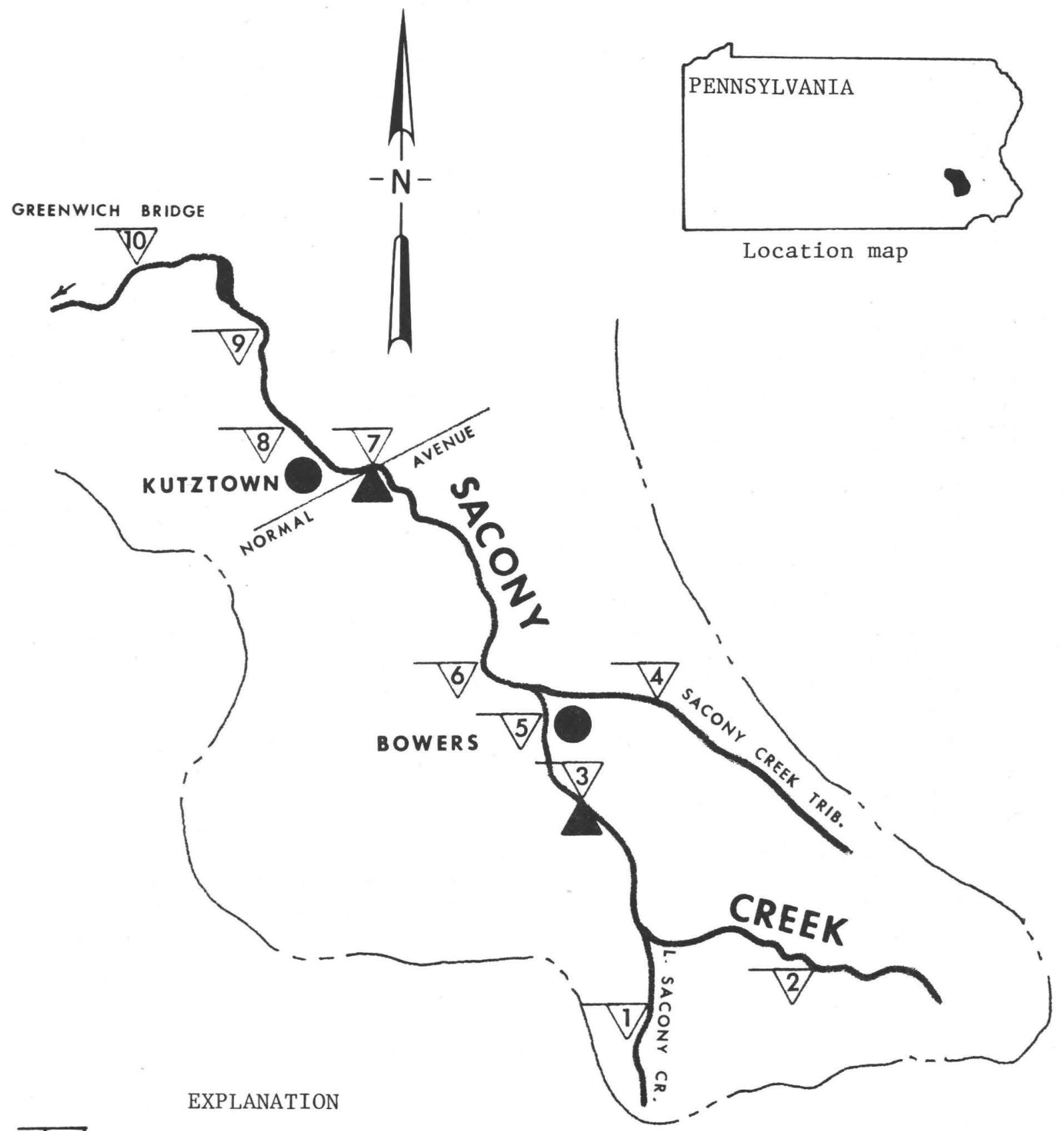

$V$

BIOLOGICAL SAMPLING STATION

GAGING STATION

TOWN

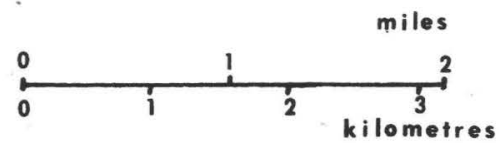

BASIN BOUNDARY

Figure 1.--Sacony Creek study area and sampling locations. 
The Sacony basin upstream from Bowers is underlain by granitic gneiss and other igneous and metamorphosed rocks. The bedrock of the 5$\mathrm{mi}(8 \mathrm{~km})$ reach downstream of Bowers is limestone and dolomite of Cambrian and Ordovician age. North and west of Kutztown the bedrock is shale and siltstone of the Martinsburg Formation of Ordovician age.

\section{Climatology}

The climate of the study area is generally characteristic of continental areas despite the proximity of the Atlantic 0cean. Prevailing air flows are westerly. The annual average temperature is $51^{\circ} \mathrm{F}\left(10.6^{\circ} \mathrm{C}\right)$ and the average precipitation is 44 in (111.8 mm) per year; about half (20 in or $50.8 \mathrm{~mm}$ ) occurs as runoff.

\section{DATA COLLECTION}

\section{Physio-chemical}

Physio-chemical studies at the 10 selected sampling stations were conducted to coincide with the biological studies so that interpretations of the aquatic ecosystem may be correlated. Field analyses consisted of the determinations of discharge, temperature, specific conductance, dissolved oxygen, and $\mathrm{pH}$. Laboratory analyses consisted of the determinations of carbonate, bicarbonate, nitrogen species, or tho and total phosphorus, sulfate, chloride, biochemical oxygen demand (BOD), and chemical oxygen demand (COD). Pesticide analyses for aldrin, DDD, DDE, DDT, dieldrin, endrin, heptachlor, lindane, chlordane, heptachlor epoxide, toxaphene, plus $\mathrm{PCB}$ and $\mathrm{PCN}$ were run on raw water samples. Analýtical work was conducted according to procedures recommended by the American Public Health Association and others (1971) or the U.S. Geological Survey (Brown and others, 1970).

\section{Biologica1}

Fecal coliform bacteria

Bimonthly water samples for fecal coliform bacteria were collected in conjunction with the physio-chemical studies. Concentrations of coliform bacteria were determined by the membrane filtration method as described by Slack and others (1973). 


\section{Benthic macroinvertebrates}

Macroinvertebrates collections were made at each of the 10 stations with a dip net of $0.210 \mathrm{~mm}$ mesh size. All available habitats were thoroughly sampled at each station until no new organisms were found. Each station was sampled with the same diligence to insure a valid comparison.

Specimens were preserved with 70 percent ethyl alcohol in the field and were later sorted, counted, and identified in the laboratory. The macroinvertebrates were identified to the family level of classification unless there were more than one genera of that family in the sample, in which case they were identified further to make that distinction evident in the result.

Following sorting, identification, and counting, the diversity index and redundancy values were computed using the method described by Wilhm and Dorris (1968).

The diversity index, (d), is a number zero or greater which expresses the degree of diversity in the population. The maximum value of (d) depends upon the total number of individuals counted and can be any positive number (Wilhm, 1970). A polluted aquatic environment usually yields a (d) value of less than 1 , and a clean aquatic environment usually has a (d) value of from 3 to 4 (Wilhm and Dorris, 1968). Redundancy ( $r$ ), which is an expression of the dominance of one or more species, can also be calculated. Redundancy is inversely proportional to the number of species; therefore, a low $(r)$ value is indicative of clean water and a high value of polluted water.

\section{Fishes}

All fish collections were accomplished with a 110 volt AC power supply designed to supply AC 60-cycle voltage from 0 to 700 volts or half-wave 60cycle pulses from 0 to 300 volts DC at 300 watts as described by Sharpe and Burkhard (1969). The voltage from the power supply was regulated to maintain a pulsed direct current output of 1.0 to 1.25 amperes that was sufficient to induce electrotaxis and subnarcosis over the range of conductivity of the water sampled.

A reach of 200 to $300 \mathrm{yds}$ (182 to $273 \mathrm{~m}$ ) of stream was electrofished at each station to determine population and biomass. The area sampled was selected to be representative of the reach being investigated. An effort was made to select a riffle-pool combination at each of the 10 sampling stations. A block net was used to enclose the upstream end of the sample area to prevent movement of fish to or from the area where no natural or man-made barrier existed.

Each area was electrofished three times. Trout population estimates were based upon the removal method proposed by Zippin (1956). All fishes collected were sorted to species, counted, and weighed. In the case of trout, scale samples were removed for determination of age and to distinguish wild from hatchery fish. Lengths were also determined for each trout. Except for game fish, representative specimens of the fishes at each station was preserved in 10 percent formaldehyde and identified in the laboratory. A11 game fish were retained in a live cage until processing and returned to the stream. 
The efficiency of electrofishing is dependent upon the size of the stream, turbidity, electrical conductivity, bottom type, stream velocity, species of . fish, and experience and alertness of the sampling personnel. As indicated by Schuck (1945), direct current electrofishing has been shown to be more efficient in sampling large fish, but also is capable of sampling up to 30 percent of the fish as small as 2 in $(50 \mathrm{~mm})$ in length.

\section{RESULTS}

\section{Physio-chemical}

In general, surface water in Sacony Creek basin is of high quality with little evidence of degradation due to cultural pollution. The water is a calciumbicarbonate type that is slightly alkaline with median $\mathrm{pH}$ values between 7.4 and 8.3 at all stations. Table 1 summarizes the median or mean concentrations of the chemical and bacterial parameters measured. Dissolved oxygen values were adequate for all forms of aquatic life and were near or slightly below saturation at all sites.

On October 1, a 24-hour dissolved-oxygen study was performed on Sacony Creek. Dissolved oxygen and temperature were measured at Normal Avenue (Station 7) every 2 hours to define the diel oxygen and temperature curves. The results of the diel study are presented in figure 2 and table 2. The curves reflect the oxygen and temperature patterns resulting from diel insolation and photosynthesis processes. Samples were also collected at all other stations between the hours of 0500 and 0630 , and again between 1500 and 1700 hours to define the anticipated maximum and minimum concentrations at each station.

Water quality is progressively altered downstream from the headwaters by the runoff and infiltration of agricultural and domestic wastes and the natural solution of minerals. From the headwaters to Sacony Creek above Kutztown (Station 6), the dissolved solids are fairly low ( 55 to $60 \mathrm{mg} / 1$ ). At Normal Avenue at Kutztown (Station 7) and downstream the carbonate rock and pollution increase the dissolved solids to more than $400 \mathrm{mg} / 1$ (milligrams per 1itre) during periods of low flow. Chloride, sulfate, phosphorus, and nitrogen (table 1) show substantial increases in concentration reaching a peak just downstream from the Kutztown sewage treatment plant (Station 9). There is no evidence that the increased nutrient and organic load stresses the assimilative capacity of the stream.

A major increase in the bicarbonate content and discharge at Normal Avenue (Station 7) indicates the intrusion of significant quantities of ground water from a limestone source. The quality of this ground water and its source(s) was not determined.

Water samples collected for analyses of the common chlorinated hydrocarbon pesticides during the September 1974, January 1975, and May 1975 samplings indicated no significant background levels of pesticides The findings concur with those of Truhlar and Reed (1975, p. 23) for other Pennsylvania streams where they found that "***base-flow samples contain little or no pesticide residues regardless of the residues present in basin soils." 
Table 1.--Median or mean concentrations of chemical and bacteriological constituents in Sacony Creek basin.

[Results in milligrams per litre, except as noted] $\underline{1} /$

\begin{tabular}{|c|c|c|c|c|c|c|c|c|c|c|c|c|c|c|c|c|c|}
\hline & & \multicolumn{12}{|c|}{ Laboratory analyses } & \multicolumn{4}{|c|}{ Field determinations } \\
\hline $\begin{array}{c}\text { Sta- } \\
\text { tion } \\
\text { No. }\end{array}$ & Station name & 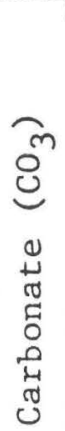 & 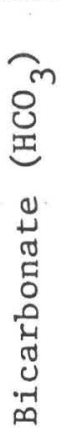 & 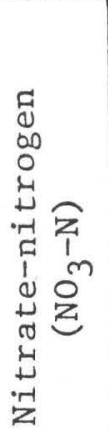 & 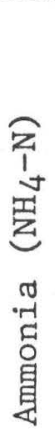 & 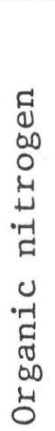 & 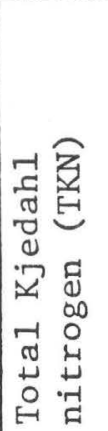 & 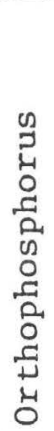 & 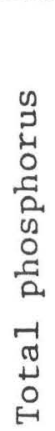 & 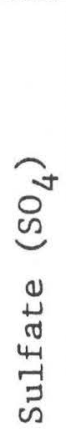 & 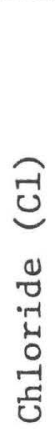 & 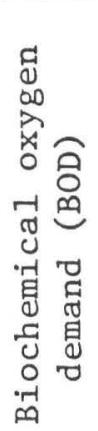 & 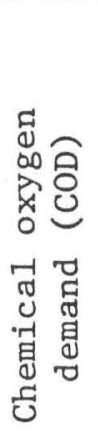 & 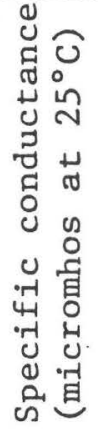 & 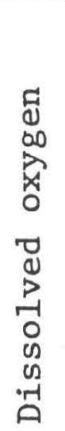 & 焉 & 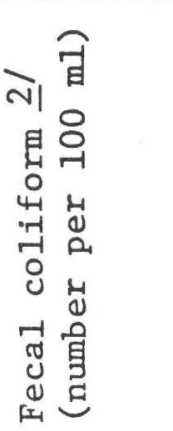 \\
\hline
\end{tabular}

$1 \quad$ Little Sacony Creek

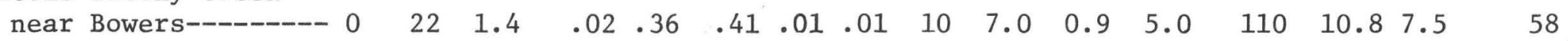

2 Sacony Creek at Sal1y Ann Furnace, near Bowers--_- $\begin{array}{lllllllllllllllll}0 & 24 & 1.3 & .03 & .42 & .44 & .01 & .01 & 12 & 3.0 & .8 & 6.0 & 100 & 11.2 & 7.5 & 55\end{array}$

3 Sacony Creek above

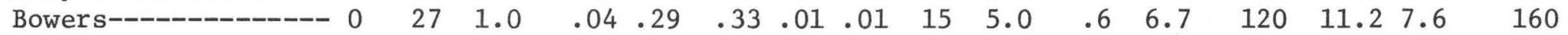

4 Sacony Creek tributary

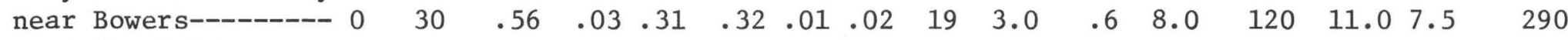

5 Sacony Creek below $\begin{array}{llllllllllllllll}\text { Bowers---1.01 } & \text { 93 } & .02 & .38 & .39 & .01 & .01 & 14 & 4.8 & 1.2 & 8.0 & 110 & 10.6 & 8.3 & 300\end{array}$

6 Sacony Creek above

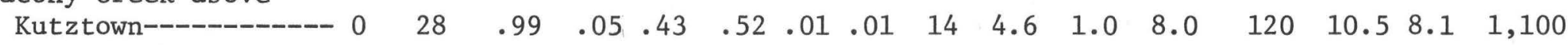

7 Sacony Creek at Normal

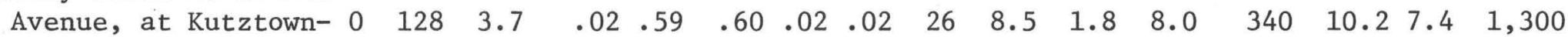

8 Sacony Creek at

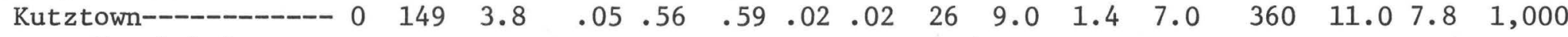

9. Sacony Creek below

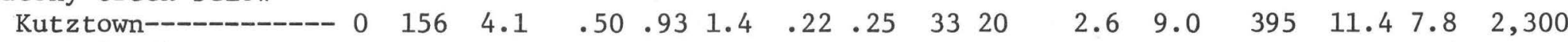

10 Sacony Creek at Greenwich Bridge,

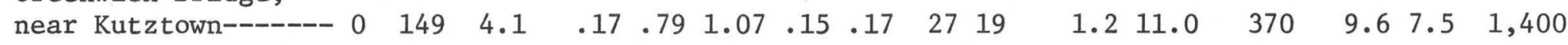



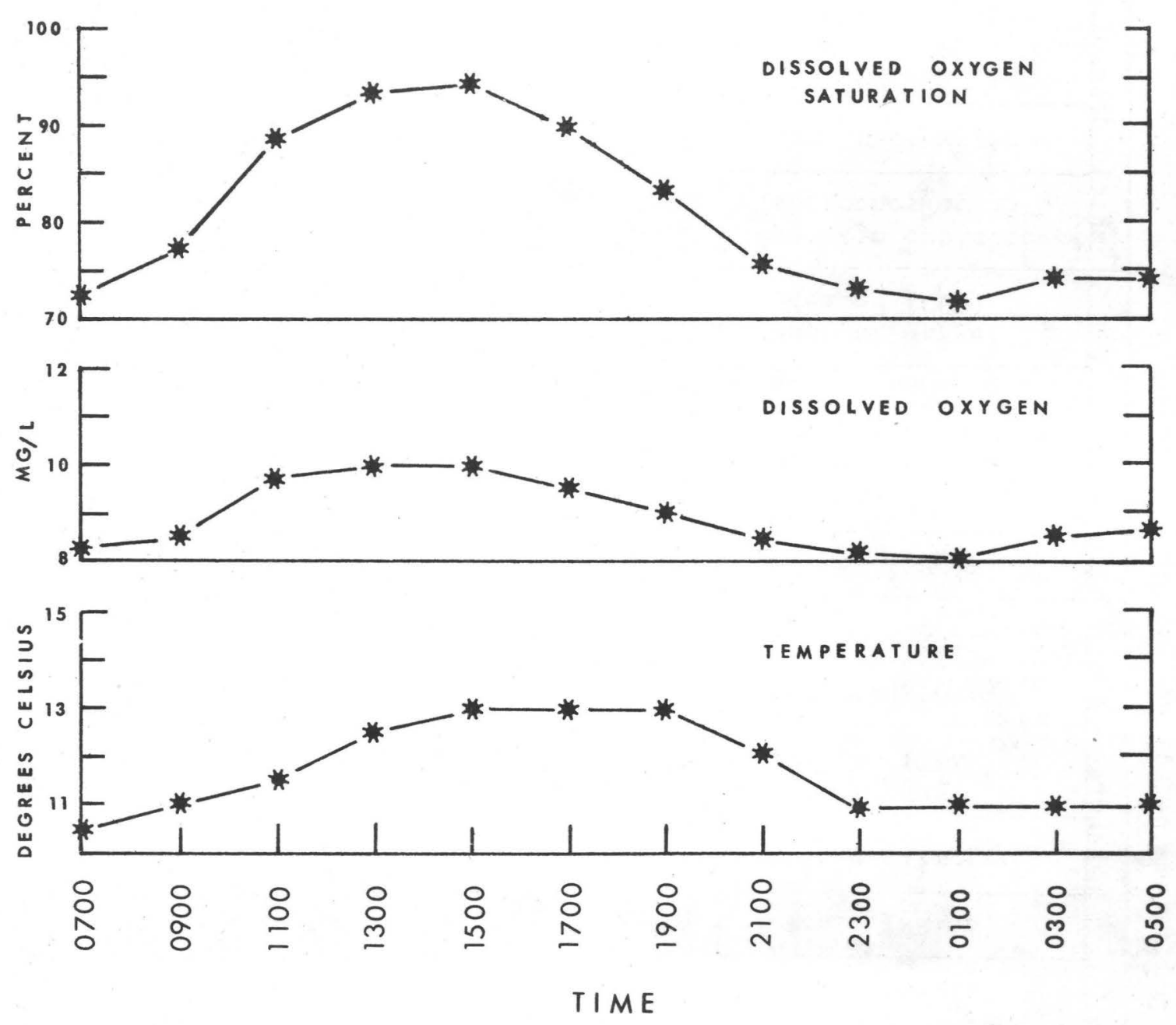

Figure 2.--Diel fluctuation in dissolved oxygen and temperature in Sacony Creek (Station 2) on October 1-2, 1974. 
Table 2.--Results of maximum and minimum dissolved-oxygen study in Sacony Creek.

\begin{tabular}{|c|c|c|c|c|c|}
\hline $\begin{array}{l}\text { Sta- } \\
\text { tion } \\
\text { No. }\end{array}$ & Station name & Time & $\begin{array}{l}\text { Disso } \\
\mathrm{mg} / \mathrm{I}\end{array}$ & $\begin{array}{l}\frac{\text { ed oxygen }}{\text { Percent }} \\
\text { saturation }\end{array}$ & $\begin{array}{l}\text { Water } \\
\text { temperature } \\
\left({ }^{\circ} \mathrm{C}\right)\end{array}$ \\
\hline 1 & $\begin{array}{l}\text { Little Sacony Creek } \\
\text { near Bowers-- }\end{array}$ & $\begin{array}{l}0610 \\
1610\end{array}$ & $\begin{array}{l}9.8 \\
9.9\end{array}$ & $\begin{array}{l}87 \\
89\end{array}$ & $\begin{array}{l}10 \cdot 5 \\
11.0\end{array}$ \\
\hline 2 & $\begin{array}{l}\text { Sacony Creek at Sally } \\
\text { Ann Furnace, near } \\
\text { Bowers-- }\end{array}$ & $\begin{array}{l}0605 \\
1520\end{array}$ & $\begin{array}{l}9.7 \\
9.8\end{array}$ & $\begin{array}{l}85 \\
90\end{array}$ & $\begin{array}{l}10.0 \\
12.0\end{array}$ \\
\hline 3 & $\begin{array}{l}\text { Sacony Creek above } \\
\text { Bowers---- }\end{array}$ & $\begin{array}{l}0600 \\
1510\end{array}$ & $\begin{array}{l}9.7 \\
9.8\end{array}$ & $\begin{array}{l}85 \\
92\end{array}$ & $\begin{array}{l}10.0 \\
13.0\end{array}$ \\
\hline 4 & $\begin{array}{c}\text { Sacony Creek tributary } \\
\text { near Bowers }\end{array}$ & $\begin{array}{l}0550 \\
1620\end{array}$ & $\begin{array}{l}9.8 \\
9.8\end{array}$ & $\begin{array}{l}86 \\
92\end{array}$ & $\begin{array}{l}10.0 \\
13.0\end{array}$ \\
\hline 5 & $\begin{array}{l}\text { Sacony Creek below } \\
\text { Bowers--- }\end{array}$ & $\begin{array}{l}0545 \\
1625\end{array}$ & $\begin{array}{l}9.7 \\
9.9\end{array}$ & $\begin{array}{l}85 \\
93\end{array}$ & $\begin{array}{l}10.0 \\
13.0\end{array}$ \\
\hline 6 & $\begin{array}{l}\text { Sacony Creek above } \\
\text { Kutztown---- }\end{array}$ & $\begin{array}{l}0540 \\
1630\end{array}$ & $\begin{array}{r}7.6 \\
10.0\end{array}$ & $\begin{array}{l}67 \\
95\end{array}$ & $\begin{array}{l}10.5 \\
13.5\end{array}$ \\
\hline 7 & $\begin{array}{c}\text { Sacony Creek at Norma } 1 \\
\text { Avenue, at Kutztown }\end{array}$ & $\begin{array}{l}0530 \\
1700\end{array}$ & $\begin{array}{l}8.2 \\
9.9\end{array}$ & $\begin{array}{l}73 \\
93\end{array}$ & $\begin{array}{l}10.5 \\
13.0\end{array}$ \\
\hline 8 & $\begin{array}{l}\text { Sacony Creek at } \\
\text { Kutztown-- }\end{array}$ & $\begin{array}{l}0525 \\
1645\end{array}$ & $\begin{array}{l}8.6 \\
9.6\end{array}$ & $\begin{array}{l}77 \\
91\end{array}$ & $\begin{array}{l}11.0 \\
13.5\end{array}$ \\
\hline 9 & $\begin{array}{l}\text { Sacony Creek below } \\
\text { Kutztown-- }\end{array}$ & $\begin{array}{l}0520 \\
1650\end{array}$ & $\begin{array}{l}8.6 \\
9.6\end{array}$ & $\begin{array}{l}77 \\
91\end{array}$ & $\begin{array}{l}11.0 \\
13.5\end{array}$ \\
\hline 10 & $\begin{array}{l}\text { Sacony Creek at } \\
\text { Greenwich Bridge, } \\
\text { near Kutztown------ }\end{array}$ & $\begin{array}{l}0515 \\
1700\end{array}$ & $\begin{array}{l}7.2 \\
9.1\end{array}$ & $\begin{array}{l}66 \\
86\end{array}$ & $\begin{array}{l}12.0 \\
13.5\end{array}$ \\
\hline
\end{tabular}




\section{Biological}

Fecal coliform bacteria

Fecal coliform bacteria concentrations increased progressively from the headwaters toward the downstream sampling station. As with the various chemical constituents, the concentrations of bacteria are affected by the runoff and infiltration of natura1, agricultura1, and domestic pollutants. Table 1 shows the geometric mean concentrations of fecal coliform bacteria reaching a peak just downstream of the Kutztown sewage treatment plant.

\section{Benthic macroinvertebrates}

The results of the macroinvertebrate study support other findings that the waters of Sacony Creek are, in general, clean and capable of supporting a biologically healthy population of organisms. The macroinvertebrates identified and their numbers are listed in table 3.

Diversity (d), listed in table 4, ranged from a low of 2.44 at Station 1 to a high of 3.46 at Station 7 , while redundancy values ( $r$ ) ranged from 0.14 at Station 4 to 0.38 at Station 1 . The diversity (d) and redundancy $(r)$ values tend to support other biological and chemical data that indicate the waters to be of good quality. Variability in (d) and ( $r$ ) between stations may, in part, be explained by inadequate sampling. As pointed out by Wilhm (1970), five samples are sometimes required in order to obtain a consistent value.

The physical characteristics of Sacony Creek change from the upper to the lower reaches. The upper stations are characterized by swift, shallow, turbulent waters, with many riffles and few pools. Bottom materials are boulders, sand and gravel with practically no aquatic vegetation. The lower stations have slower, deeper water with a combination of riffle and pool areas. Bottom materials here are a mixture of cobbles, grave1, sand, and mud with abundant rotted aquatic vegetation. The available habitat at the lower stations is more favorable for a diverse population.

The relatively low (d) value and high ( $r$ ) value of Station 1 is probably due to the limited habitat necessary to support a large number of species. Al1 chemical investigations verify that the water quality at Station 1 is equal to or superior to that at the other stations samples.

\section{Fishes}

The results of the fish population data are summarized in table 5. A total of 8,291 individual specimens, representing 29 species and weighing 820 lbs $(372 \mathrm{~kg})$, were collected at the 10 stations sampled in the Sacony Creek basin. The number of species ranged from 4 at Little Sacony Creek (Station 1) to 20 at Greenwich Bridge (Station 10). 
Table 3.--Population composition of benthic macroinvertebrates in Sacony Creek, September 24-27, 1974.

Common name

Segmented Worms

Leeches

Joint Footed Animals

Aquatic Arthropods

Scuds, Sideswimmers

Aquatic Sowbugs

Crayfish

Insects

Mayflies

Dragonflies, Damse1flies

True Bugs

Back Swimmers

Dobsonflies, Hellgrammites, Fishflies, Alderflies

Caddis F1ies
Phylum

Class

$\underline{\text { Order }}$

Annelida

Hirudinea Rhynchobdellida

Arthropoda

Crustacea

Amphipoda

Isopoda

Decapoda

Insecta

Ephemeroptera
Odonata

Hemoptera

Megaloptera

Trichoptera 
Table 3.--Population composition of benthic macroinvertebrates in Sacony Creek, September 24-27, 1974.

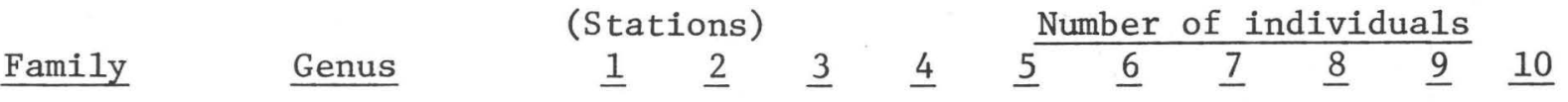

G1ossiphoniidae

$2 \longdiv { 5 } \quad 1 \quad 1$

Gammaridae

Ase11idae

Astacidae

Heptageniidae

Baetidae

Ephemeridae

Coenagrioridae

Gomphidae

Agrianidae

Notonectidae

Corydalidae

Sialidae

Hydropsychidae

Philopotamidae

Hydroptilidae

Limnephilidae

Rhyacophilidae
Gammarus

Ase11us
$39 \quad 53$

9

$39 \quad 142$

$11 \quad 75 \quad 11$

phemerella

Habrophlebia

Potamanthus $\begin{array}{llll}3 & 3 & 1 & \\ & 3 & 1 & 3\end{array}$ $\begin{array}{llll}12 & 38 & 9 & 1\end{array}$

$\begin{array}{lllll}1 & 11 & 8 & 10 & 5\end{array}$

Corydalus

Chauliodes

Sialis

1

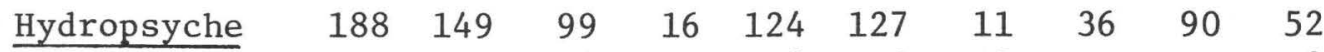

$\begin{array}{llllllll}\text { Chimarra } & 71 & 28 & 34 & 4 & 2 & 2 & 2\end{array}$

3.

Agapetus

$\begin{array}{llll}\text { Rhyacophila } & 4 & 7 & 11\end{array}$

$13 \quad 11$

93

1 
Table 3.--Population composition of benthic macroinvertebrates in Sacony Creek, September 24-27, 1974--continued.

Common name

Aquatic Caterpillars

Beetles

Water Pennies

Predaceous Diving Beetles

Water Scavenger Beetles

F1ies

Black F1ies

Midges

Crane Flies

Horse F1ies

Snipe Flies

Moth Flies

Soldier Flies

Shore Flies

Stoneflies

Mollusks

Snails, Limpets

Clams, Mussels
Phylum

$\underline{\text { Class }}$

Order

Lepidoptera

Coleoptera

Diptera

Plecoptera

Mollusca

Gastropoda Pulmonata

Pelecypoda 
Table 3.--Population composition of benthic macroinvertebrates in Sacony Creek, September 24-27, 1974--continued.

$\underline{\text { Family }}$

Pyralididae

Unidentified

Psephenidae

Elmidae

Dytiscidae

Dryopidae

Hydrophilidae

Unidentified

Simuliidae

Tendipedidae

Tipulidae

Tabanidae

Rhagionidae

Psychodidae

Stratiomyiidae

Ephydridae

Pteronarcidae

Peltoperlidae

Perlidae

Pteronorcys

Peltoper1a

Acroneuria

Total number of types
Total number of ind.
Sphaeriidae

Total number of types
Total number of ind.

\section{Ferrissia \\ Physa \\ Heliosoma \\ Planorbidae \\ Ancylidae \\ Physidae}

(Stations)

Number of individuals

$\begin{array}{llllllllll}1 & \underline{2} & \underline{3} & \underline{4} & \underline{5} & \underline{6} & \underline{7} & \underline{8} & \underline{9} & \underline{10}\end{array}$

1

$\begin{array}{rrrrrrrrrr}1 & 1 & 7 & & 13 & 8 & 8 & & 1 & 7 \\ 14 & 35 & 2 & 4 & 13 & 47 & 4 & 13 & 12 & 15 \\ & 2 & & & & & 1 & 1 & & \\ 2 & 2 & & & & 2 & & & & \end{array}$

$\begin{array}{rrrllrrrrr}24 & 12 & 6 & & & 4 & 1 & 3 & 3 & \\ 11 & 37 & 71 & & 16 & 45 & 39 & 81 & 64 & 52 \\ 2 & 15 & 1 & 1 & & 6 & & & 3 & 2 \\ & & 4 & 1 & & & 2 & 5 & 1 & 1 \\ & 7 & 9 & & 5 & 1 & & & 1 & 3 \\ & 1 & & & & & & & & \end{array}$

1
$14 \quad 23$

385

$\begin{array}{rrrr} & 8 & 3 & \\ 11 & 32 & 1 & 1 \\ & 33 & 15 & \end{array}$

123
1

$\begin{array}{llllll}1 & 1 & 2 & 2 & 8 & 7 \\ & & & & & 1\end{array}$

$\begin{array}{llll}1 & 2 & 5 & 1\end{array}$

$\begin{array}{llllllll}25 & 15 & 19 & 18 & 19 & 20 & 18 & 19\end{array}$

$\begin{array}{llllllll}428 & 47 & 278 & 311 & 130 & 283 & 276 & 199\end{array}$ 
Table 4.--Diversity (d) and redundancy (r) of Sacony Creek benthos, September 24-27, 1974.

$\begin{array}{cccc}\text { Station } & \text { Station name } & \text { Diversity } & \text { Redundancy } \\ \text { no. } & & \text { (d) } & \text { ( } \mathrm{r} \text { ) }\end{array}$

$1 \quad$ Little Sacony Creek near Bowers---- $\quad 2.44 \quad 0.38$

2 Sacony Creek at Sally Ann Furnance, near Bowers-- 3.44

3 Sacony Creek above Bowers-------- $\quad 3.35 \quad .29$

4 Sacony Creek tributary near Bowers-- $\quad 3.13 \quad .14$

5 Sacony Creek below Bowers-------- $\quad 2.86 \quad .37$

6 Sacony Creek above Kutztown------ $\quad 2.73$

7 Sacony Creek at Normal Avenue, at Kutztown- $3.46 \quad .24$

8 Sacony Creek at Kutztown-- 3.33

9 Sacony Creek below Kutztown------ $\quad 2.92 \quad .32$

10 Sacony Creek at Greenwich Bridge, near Kutztown-- 32 
Table 5.--Number and standing crop per acre of fish inhabiting selected reaches of Sacony Creek basin.

[Number per acre with corresponding pounds per acre in parentheses]

Common name

Brook trout

Brown trout

Rainbow trout

White sucker

Blacknose dace

Longnose dace

Northern creek chub

Cutlips minnow

Common shiner

Common sunfish 1 /

Johnny darter

Yellow perch

Satinfin shiner

Bluntnose minnow

Spottail minnow

Eastern madtom

Largemouth bass

E. banded killifish

E. creek chubsucker

Golden shiner

Redfin pickere1

Brown bullhead

Rock bass

Carp

$\mathrm{N}$ swallowtail shiner

Fallfish

Smallmouth bass

Black crappie

American eel
Scientific name

Salvelinus fontinalis

Salmo trutta

Salmo gairdneri

Catostomus commersoni

Rhinichthys atratulus

Rhinichthys cataractae

Semotilus atromaculatus

Exoglossum maxillingua

Notropis cornutus

Lepomis sp.

Etheostoma nigrum

Perca flavescens

Notropis analostanus

Pimephales notatus

Notropis hudsonius

Schilbeodes insignis

Micropterus salmoides

Fundulus diaphanus

Erinyzon oblongus

Notemigonus crysoleucas

Esox americanus

Ictalurus nebulosus

Ambloplites rupestris

Cyprinus carpio

Notropis procne

Semotilus corporalis

Micropterus dolomieui

Pomoxis nigromaculatus

Anguilla rostrata

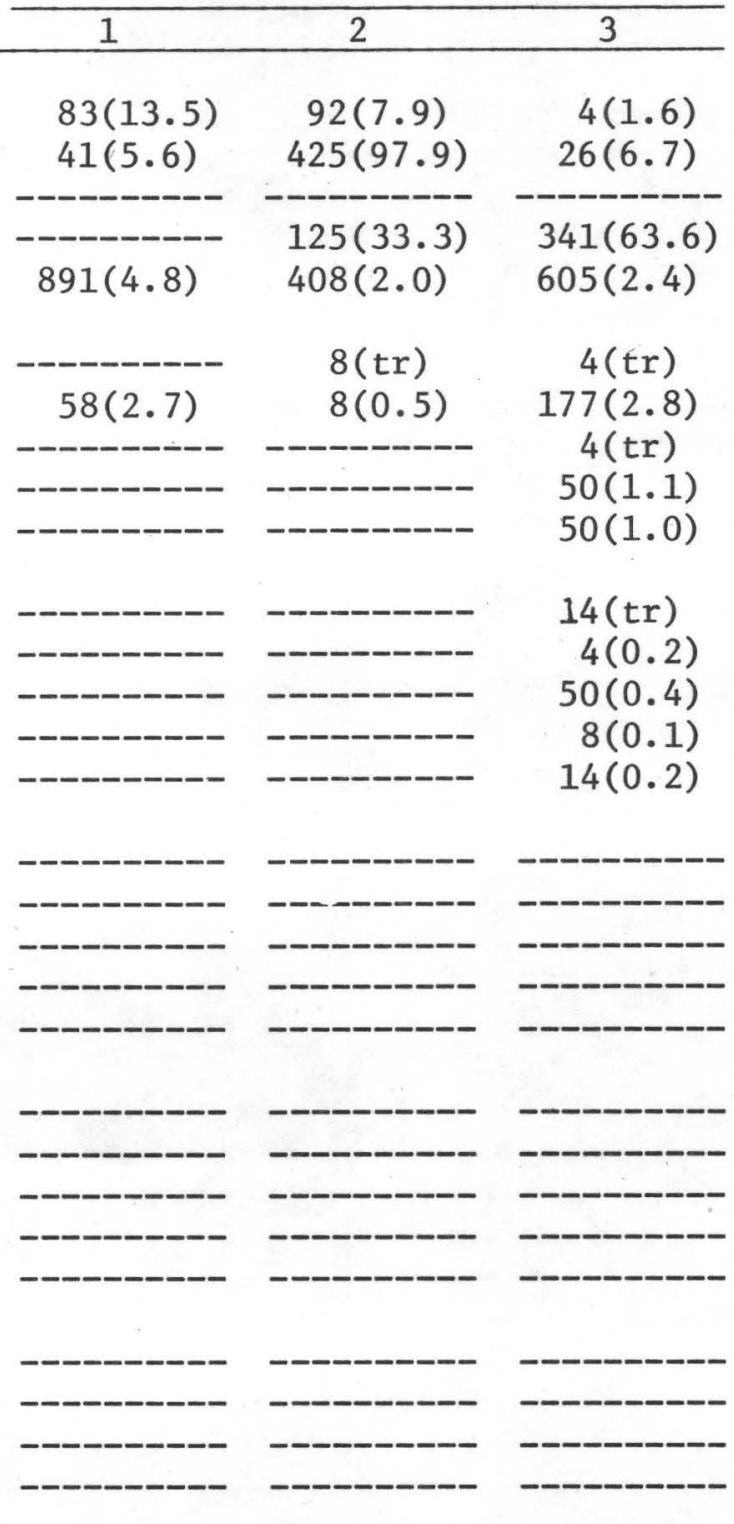

$\begin{array}{ccc}4 & 6 & 15 \\ 124(19.1) & 517(105.8) & 30(8.3) \\ 949(7.5) & 549(35.8) & 1321(71.8) \\ 1073(26.6) & 1066(141.6) & 1351(80.1)\end{array}$
$1066(141.6)$
Number of species

Total trout

Total non-trout

Grand tota1

1/ Includes Lepomis auritus, L. gibbosus, and hybreds. 
Table 5.--Number and standing crop per acre of fish inhabiting selected reaches of Sacony Creek basin.

[Number per acre with corresponding pounds per acre in parentheses]

Stream station number

\begin{tabular}{|c|c|c|c|c|c|c|}
\hline 4 & 5 & 6 & 7 & 8 & 9 & 10 \\
\hline --ー--ー---- & ----------- & ----------- & $14(2.1)$ & $2(0.3)$ & $1(t r)$ & ----- \\
\hline -- & $4(2.2)$ & - & $14(2.8)$ & - & - & --- \\
\hline $1400(51.4)$ & $881(76.9)$ & $2019(154.8)$ & $2135(525.7)$ & $1698(291.8)$ & $1085(352.2)$ & $850(56.0)$ \\
\hline $1500(0.5)$ & $481(2.2)$ & $2571(17.5)$ & $14(0.1)$ & $56(0.2)$ & $131(0.8)$ & $317(2.2)$ \\
\hline & $4(\operatorname{tr})$ & $5(\mathrm{tr})$ & ----- & $2(t r)$ & $4(\operatorname{tr})$ & $397(4.0)$ \\
\hline $650(4.4)$ & $231(1.0)$ & $152(4.0)$ & $3(0.5)$ & --- & $26(1.8)$ & $130(2.6)$ \\
\hline & $12(0.2)$ & $14(0.3)$ & $3(0.1)$ & ---------- & $13(0.5)$ & $587(6.8)$ \\
\hline $267(1.0)$ & $85(0.3)$ & $180(3.3)$ & $49(1.8)$ & $11(0.3)$ & $7(0.2)$ & $103(2.6)$ \\
\hline & $219(5.2)$ & $171(5.1)$ & $132(5.1)$ & $50(1.8)$ & $72(2.6)$ & $130(4.0)$ \\
\hline $200(0.8)$ & $31(0.2)$ & $52(0.3)$ & $305(1.5)$ & $308(1.5)$ & $112(1.5)$ & $113(0.5)$ \\
\hline------- & $669(3.5)$ & $614(3.1)$ & $41(0.3)$ & $29(0.2)$ & $10(0.1)$ & $47(0.5)$ \\
\hline $17(t r)$ & $519(2.4)$ & $486(3.1)$ & $57(0.4)$ & $21(0.1)$ & $44(0.2)$ & $102(1-10$ \\
\hline & $204(2.9)$ & & & & & मU> \\
\hline--- & $27(0.4)$ & $129(2.7)$ & $5(0.2)$ & & & $77(2.5)$ \\
\hline$\cdots-$ & $4(0.1)$ & ------- & $5(0.1)$ & $15(0.4)$ & $18(0.7)$ & $7(0.2)$ \\
\hline- & $4(21)$ & $200(1.0)$ & $105(9.5)$ & $65(1.1)$ & $19(1.8)$ & --_------ \\
\hline-- & - & - & $138(2.2)$ & $42(1.6)$ & $141(3.6)$ & \\
\hline - & $\ldots$ & $-\ldots$ & $273(4.9)$ & $79(1.4)$ & $10(0.8)$ & \\
\hline - & ---- & $-\cdots$ & $3(0.6)$ & & & $17(3.8)$ \\
\hline-- & - & - & --- & - & $12(1.5)$ & $40(4.0)$ \\
\hline------ & - & - & -- & $9(6.6)$ & $\begin{array}{l}16(3.3) \\
69(0.2)\end{array}$ & $\begin{array}{r}10(1.0) \\
793(3.7)\end{array}$ \\
\hline & & & & & & $70(3.7)$ \\
\hline -- & -- & ----------- & ---------- & ----------- & --------- & $13(0.2)$ \\
\hline ----- & ------ & -------- & --------- & ------ & -------- & $3(0.3)$ \\
\hline & & & & & & \\
\hline 6 & 16 & 14 & 19 & 17 & 19 & 20 \\
\hline 0 & $4(2.2)$ & 0 & $28(4.9)$ & $2(0.3)$ & $1(\mathrm{tr})$ & 0 \\
\hline $4034(58.1)$ & $3371(95.3)$ & $6622(196.2)$ & $3352(553.8)$ & $2446(307.6)$ & $1789(372.0)$ & $3807(106.5)$ \\
\hline 4034(58.1) & $3375(97.5)$ & $6622(196.2)$ & $3380(558.7)$ & $2448(307.9)$ & $1790(372.0)$ & $3807(106.5)$ \\
\hline
\end{tabular}


Three species of fish found at most stations were the white sucker (Catostomus commersoni), blacknose dace (Rhinichthys atratulus), and northern creek chub (Semotilus atromaculatus). The headwater stations (1 and 2) contained the fewest species but the largest trout populations. A few species such as the American eel (Anguilla rostrata), smallmouth bass (Micropterus dolomieui), fallfish (Semotilus corporalis), rainbow trout (Salmo gairdneri), and black crappie (Pomoxis nigromaculatus) were found in small numbers and at few sites. In general, a greater diversity of species was found lower in the watershed. This is due to a greater diversity of habitat and warmer water.

The standing crop of fishes based upon actual collections ranged from a minimum of $26.6 \mathrm{lbs} /$ acre $\left(12.1 \mathrm{~kg} / \mathrm{hm}^{2}\right.$ ) at Little Sacony Creek (Station 1) to $558.7 \mathrm{lbs} /$ acre $\left(253.4 \mathrm{~kg} / \mathrm{hm}^{2}\right.$ ) at Normal Avenue (Station 7). The bulk of the standing crop from Sacony Creek above Bowers to Greenwich Bridge (Stations 3 to 10) is due to the large population of white suckers. In fact, the white sucker composed from 53 to 95 percent of the weight at Stations 3 through 10. Species diversity and standing crop are summarized in figure 3.

Trout inhabited seven of the 10 sampling stations. brook trout (Salvelinus fontinalis) were found at six stations, brown trout (Salmo trutta) at four, and rainbow trout (Salmo gairdneri) at one. Trout dominated the standing crop in the headwaters (Stations 1 and 2) and were a significant segment of the standing crop downstream to Bowers (Station 3). Downstream from Bowers, trout become an incidental species and do not successfully compete for dominance with several species of minnows and the white sucker.

No trout were revealed at Stations 4, 6, and 10. The lack of suitable habitat is believed the reason for trout not being in the sample at Station 6 , as several brook trout were observed about half a mile downstream of the sample area. Stations 4 and 10 contained suitable habitat for trout, so their absence in the sample must be attributed to other causes. It was observed that Stations 4 and 10 contained very few adult fish and lower biomass than expected, suggesting periodic environmenta1 stress.

Age Composition of Trout Populations.--The population of brook and brown trout were separated into age groups according to the number of annuli or year marks as shown in tables 6 and 7. Brook trout older than age-group I were rare and none were found older than age-group II. Brown trout were found up to age-group IV, with on1y 8 percent older than age-group II. These data correspond closely with that of McFadden and Cooper (1961) for other Pennsylvania streams.

Hatchery fish were distinguished from wild fish primarily by the growth patterns of the scales. Wild fish characteristically have a slower rate of growth, particularly during the first year. They also have a lower incidence of scale regeneration and their gross external appearance is usually unlike a hatchery fish. 


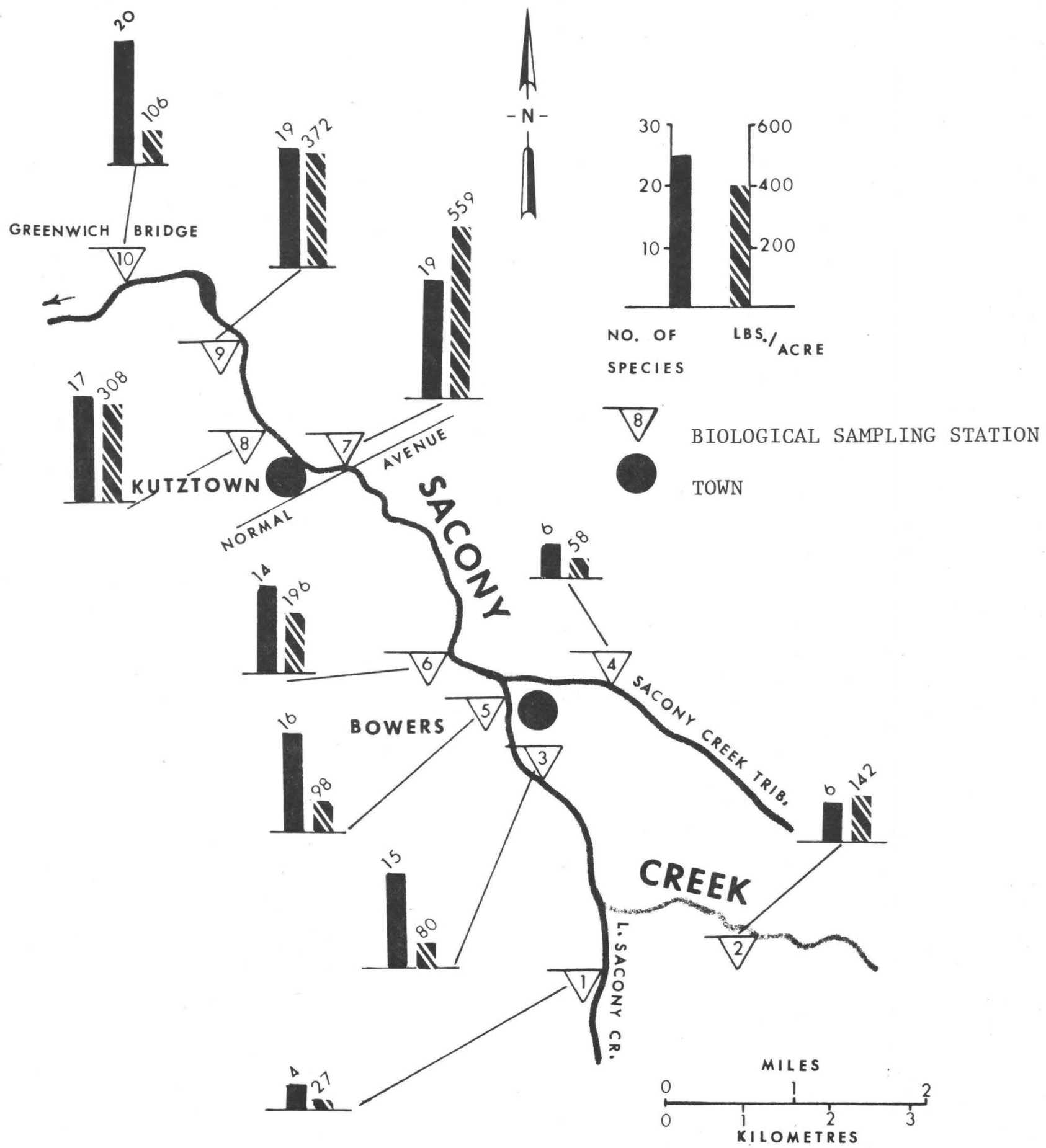

Figure 3.--Species diversity and standing crop of fishes in Sacony Creek basin. 
Table 6.--Length-age frequency of Sacony Creek brook trout.

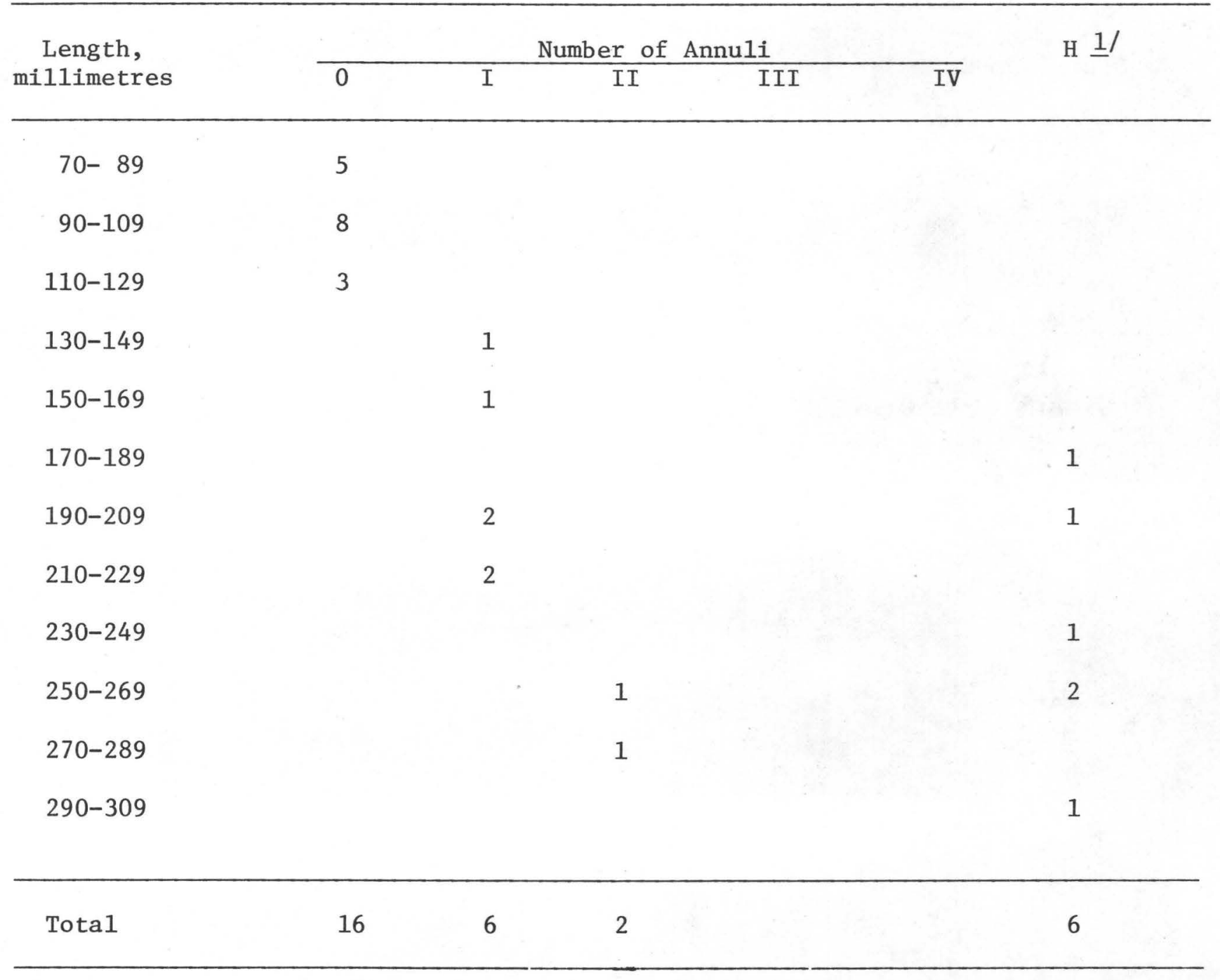

1/ Fish of hatchery origin. 
Table 7.--Length-age frequency of Sacony Creek brown trout.

\begin{tabular}{|c|c|c|c|c|c|c|}
\hline \multirow{2}{*}{$\begin{array}{l}\text { Length, } \\
\text { millimetres }\end{array}$} & \multicolumn{5}{|c|}{ Number of Annuli } & \multirow[b]{2}{*}{$\mathrm{H}^{1 /}$} \\
\hline & 0 & I & II & III & IV & \\
\hline $70-89$ & 4 & & & & & \\
\hline 90-109 & 10 & & & & & \\
\hline $110-129$ & 3 & & & & & \\
\hline \multicolumn{7}{|l|}{$130-149$} \\
\hline $150-169$ & & 2 & & & & \\
\hline $170-189$ & & 20 & & & & \\
\hline $190-209$ & & 3 & & & & \\
\hline $210-229$ & & 2 & 4 & & & 2 \\
\hline $230-249$ & & & 4 & & & 3 \\
\hline $250-269$ & & & 2 & & & 1 \\
\hline \multicolumn{7}{|l|}{$270-289$} \\
\hline $290-309$ & & & & 2 & & 1 \\
\hline $310-329$ & & & & 1 & & \\
\hline $330-349$ & & & & 1 & & \\
\hline $350-359$ & & & & & & 1 \\
\hline \multicolumn{7}{|l|}{$370-389$} \\
\hline \multicolumn{7}{|l|}{$390-409$} \\
\hline \multicolumn{7}{|l|}{$410-429$} \\
\hline $430-449$ & & & & & 1 & \\
\hline
\end{tabular}

1/ Fish of hatchery origin. 
Growth of brown trout in Sacony Creek is compared to the growth rate of fish reared in the Pennsylvania hatchery system (fig. 4). It takes the wild fish in Sacony Creek nearly 48 months of growth to attain the size of a 19-month old. hatchery fish. The growth rate of wild brown trout in Sacony Creek agrees favorably with that in other trout streams in Pennsylvania (McFadden and Cooper, 1961).

Both the brook trout and brown trout populations in Sacony Creek are essentially of wild origin. Although the stream is stocked with hatchery brook, brown and rainbow trout, hatchery fish composed only 20 percent of the brook trout and 12 percent of the brown trout population. The single rainbow trout found at Station 5 was of hatchery origin.

The number of wild trout in the population decreases rapidly downstream of Stations 1 and 2. Only four wild brook trout and five wild brown trout were found outside of Stations 1 and 2, indicating little migration to the lower reaches.

Estimates of Trout Populations.--The trout populations were projected by the graphical method for determining maximum likelihood estimates of population size based upon removal data as described by Zippin (1956). The estimates presented in table 8 are based upon removal data for three runs over each sample area.

Station 2 was the only location where the numbers of trout escaping capture on the second run remained high. The high probability of capture after three runs is indicated by the small difference between the captured and estimated populations at a11 stations.

Considering the relative low fertility of the headwaters, the estimated 590 trout or $119 \mathrm{lbs} /$ acre $\left(133 \mathrm{~kg} / \mathrm{hm}^{2}\right.$ ) at Station 2 is quite high and represents 77 percent of the standing crop. By comparison, McFadden and Cooper (1961) reported an average of 479 trout or $74 \mathrm{lbs}$ acre $\left(82.9 \mathrm{~kg} / \mathrm{hm}^{2}\right.$ ) for six Pennsylvania streams with wild trout populations. The high standing crop of trout at Station 2 is due to good habitat and minimal exploitation by anglers.

Efficiency of the electrofishing effort was not evaluated. However, an estimate of the proportion of the trout population sampled can be derived from table 8. As previously mentioned, only at Station 2 was a high percentage of the estimated trout population remaining after the second run. The extremely rocky streambed and poor visibility due to high velocity and turbulance are the primary reasons for the poor recovery at Station 2. In general, it is believed a high percentage of the larger fish and thus an equally high percentage of the biomass was collected at all stations. 
EXPLANATION

- SACONy CREEK MEAN LENGTH OF YEAR CLASS

BENNER SPRING HATCHERY

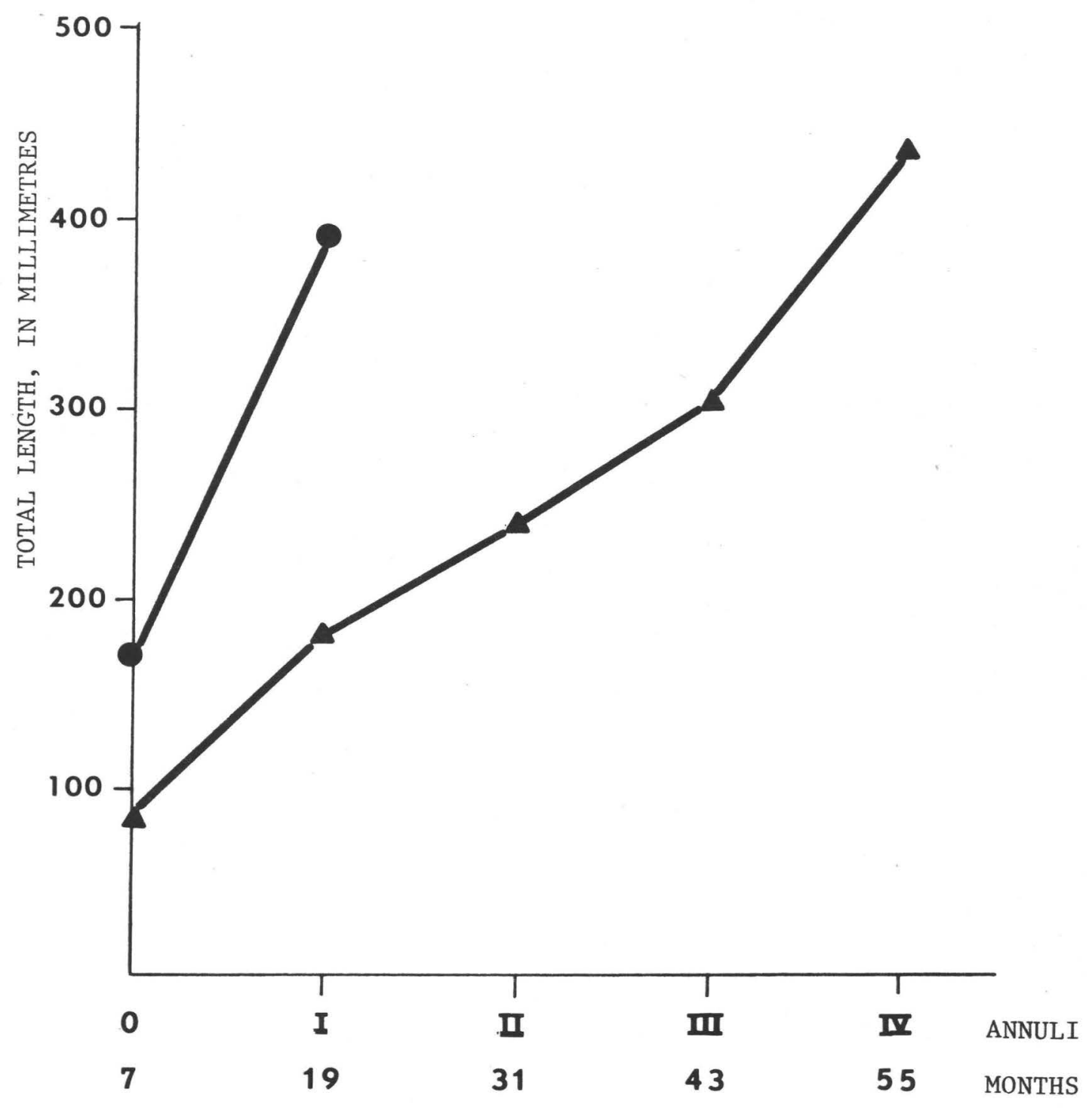

Figure 4.--Comparative Zength and age of brown trout in Sacony Creek and Benner Springs Hatchery. 
Table 8.--Population estimate of trout in selected reaches of Sacony Creek.

[Graphical method of Zippin, 1956]

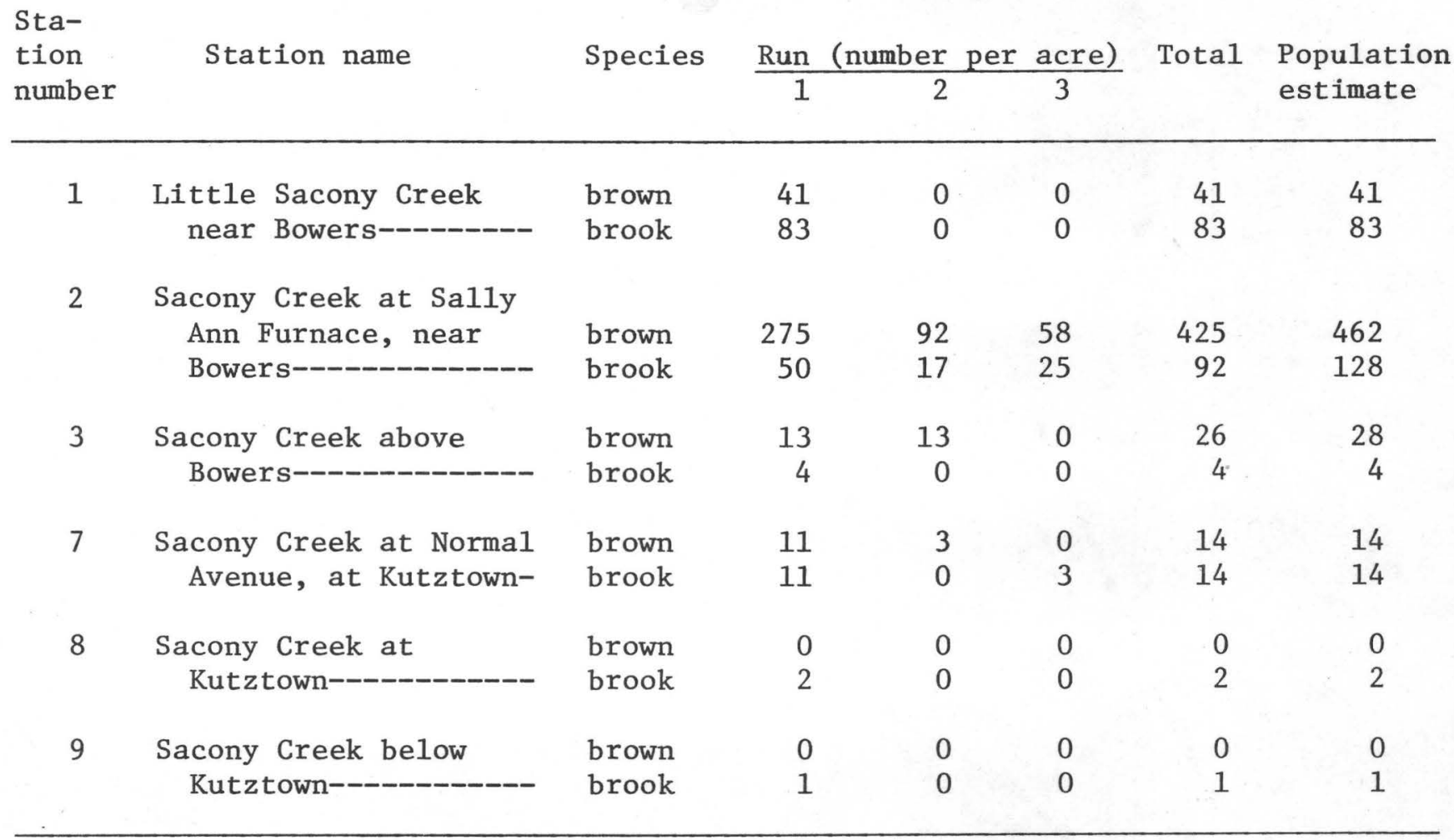


Water quality and the general health of the Sacony Creek basin was found to be good to excellent. Chemical quality indicates little degradation from cultural pollution. There is, however, a progressive increase in dissolved nutrients, oxidizable matter, and fecal coliform bacteria as the stream flows through rich farmland. Dissolved and particulate matter reach a peak in concentration immediately downstream of the Kutztown sewage treatment plant. At no point, however, is the assimilative capacity of the stream exceeded. No chlorinated hydrocarbon pesticides were found in water samples collected in September 1974, January 1975, and May 1975.

Benthic macroinvertebrate investigations concur with other findings that the waters of Sacony Creek in general, support a healthy biota. The total number of kinds of organisms at each station ranged from 14 to 25 . Clean water forms dominated the population at all stations, with mayflies, caddis flies, and midges the most numerous. Diversity (d) and redundancy ( $r$ ) indices averaged 3.07 and 0.29 and ranged from 2.44 to 3.46 and 0.14 to 0.38 , respectively. Such values support the healthy nature of the stream.

The Sacony Creek basin was found to contain a healthy fish population with 29 species represented. The number of species at any station ranged from 4 to 20 , with the headwater stations containing the fewest species. Standing crop of fishes ranged from 27 to $559 \mathrm{lb} /$ acre $\left(626 \mathrm{~kg} / \mathrm{hm}^{2}\right)$ with the bulk of the weight in trout in the headwaters and in white suckers in the lower reaches.

Trout were found to be inhabiting 7 of the 10 sampling stations but were a dominant or significant segment of the population only at the upper three stations. Those stations lacking a significant trout population contained an excess of competitive species (white sucker), lacked suitable habitat, or both. It is significant that only four wild brook trout and five wild brown trout were found downstream of Stations 1 and 2, indicating little migration to the lower reaches.

The trout population is essentially of wild origin with only 20 percent of the brook trout and 12 percent of the brown trout of hatchery origin, despite annual stocking by the Pennsylvania Fish Commission. The wild trout were found to be a short-lived fish with no brook trout and only 8 percent of the brown trout found older than age-group II ( 31 months). The growth rate of wild trout agrees favorably with other streams in Pennsylvania of similar chemical composition. 


\section{SELECTED REFERENCES}

American Fisheries Society, 1960, A 1ist of common and scientific names of fishes from the United States and Canada: Am. Fisheries Soc. Spec. Pub. no. 2, $102 \mathrm{p}$.

American Public Health Association and others, 1971, Standard methods for the examination of water and wastewater [13th ed.]: New York, Am. Public Health Assoc., 874 p.

Beyer1y, G. B., and Cooper, E. L., 1960, Growth of brown trout in selected Pennsylvania streams: Am. Fishery Soc. Trans., v. 89, no. 3, p. 255-262.

Brown, Eugene, Skougstad, M. W., and Fishman, M. J., 1970, Methods for co1lection and analysis of water samples for dissolved minerals and gases: U.S. Geo1. Survey Techniques Water-Resources Inv., book 5, chap. A1, $160 \mathrm{p}$.

Buss, Keen and Miller, Jack, 1962, The age and growth of trout in Pennsylvania in Age and growth of the Fishes in Pennsylvania: Pennsylvania Fish Comm., 2 p.

Cairns, John, Jr., and Dickson, K. L., 1971, A simple method for the biological assessment of the effects of waste discharges on aquatic bottom dwelling organisms: Water Pollution Control Federation Jour., v. 43, no. 5, p. 755-772.

Carlander, K. D., 1953, Handbook of freshwater fishery biology: Ames, Iowa State Univ. Press, 752 p.

Eddy, Samue1, 1969, How to know the freshwater fishes [2nd ed.]: Dubuque, William C. Brown, Co., 253 p.

Hubbs, C. L., and Lagler, K. F., 1958, Fishes of the Great Lakes region: Cranbrook Inst. Sci Bu11. no. 26, 186 p.

Lagler, K. F., 1973, Freshwater fishery biology [2nd ed.]: Dubuque, William C. Brown, Co., 421 p.

McFadden, J. T., and Cooper, E. L., 1961, An ecological comparison of six populations of brown trout (Salmo trutta): Am Fishery Soc. Trans., v. 91, no. 1. p. 53-62.

Pennak, R. W., 1953, Fresh-water invertebrates of the United States: New York, The Ronald Press Co, 769 p.

Schuck, H. A., 1945, Survival, population density, growth and movements of wild brown trout in Crystal Creek: Am. Fishery Soc. Trans., v. 73, p. 209-230.

Sharpe, F. P., and Burkhard, W. T., 1969, A lightweight backpack high voltage electrofishing unit: U.S. Bur. Sport Fisheries and Wildlife Resources Pub. no. 78,8 p. 
Slack, K. V., Averett, R. C., Greeson, P. E., and Lipscomb, R. G., 1973, Methods for collection and analysis of aquatic biological and microbiological samples: U.S. Geo1. Survey Techniques Water-Resources Inv., book 5, chap. A4, 165. p.

Truhlar, J. F., and Reed, L. A., 1975, Occurrence of pesticide residues in four streams draining different land-use areas in Pennsylvania: U.S. Geo1. Survey Water Resources Inv. 6-75, 23 p.

U.S. Weather Bureau, 1974 and 1975, Climatological Data-Pennsylvania: U.S. Dept. of Commerce.

Wilhm, J. L., 1970, Range of diversity index in benthic macroinvertebrate populations: Water Pollution Control Federation Jour., v. 42, no. 5, pt. 2 .

Wilhm, J. L., and Dorris, T. C., 1968, Biological parameters for water quality criteria: Bioscience, v. 18, no. 6, p. 477-480.

Zippin, Calvin, 1956, An evaluation of the removal method of estimating animal populations: Biometrics, v. 12, p. 163-189. 
Table 9.--Water-quality determinations in Sacony Creek basin.

[Results in milligrams per litre except as noted]

\begin{tabular}{|c|c|c|c|c|c|c|c|c|c|c|}
\hline \multirow[b]{2}{*}{ Date } & \multirow[b]{2}{*}{ Time } & \multicolumn{9}{|c|}{ Laboratory analyses } \\
\hline & & 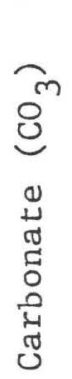 & 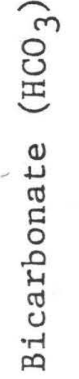 & 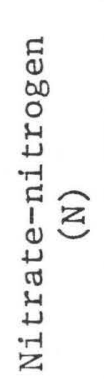 & 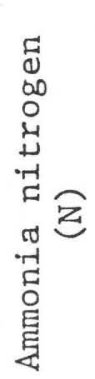 & 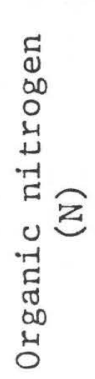 & 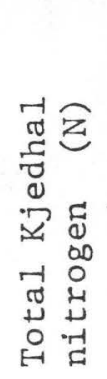 & 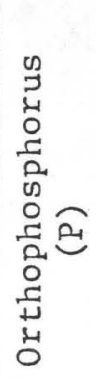 & 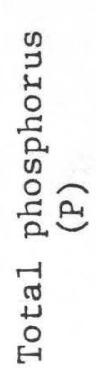 & 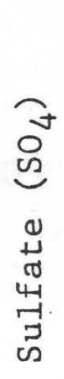 \\
\hline
\end{tabular}

Little Sacony Creek near Bowers, Pa. (Station 1)

$\begin{array}{rrrrrrrrrrr}7-31-74 & 1435 & 0 & 31 & 1.4 & 0 & 0 & 0 & 0 & 0 & 10 \\ 9-27-74 & 0930 & 0 & 29 & 2.1 & .06 & .45 & .57 & .02 & .03 & 9.7 \\ 11-14-74 & 1200 & 0 & 24 & 1.4 & .08 & .35 & .43 & .01 & .01 & 27 \\ 1-22-75 & 1400 & 0 & 17 & 1.6 & .02 & .36 & .38 & .01 & .02 & 8.6 \\ 3-12-75 & 1320 & 0 & 14 & 1.3 & .02 & .39 & .41 & .01 & .01 & 14 \\ 5-14-75 & 1100 & -- & -- & --- & ---- & ---- & ---- & -0 & ---- & -1 .- \\ 5-21-75 & 1100 & 0 & 22 & 1.2 & 0 & 0 & 0 & 0 & 0 & 12\end{array}$

Sacony Creek at Sally Ann Furnance, near Bowers, Pa. (Station 2)

$\begin{array}{rrrrrrrrrrr}7-31-74 & 1315 & 0 & 30 & 1.3 & ---- & ---- & ---- & --- & ---- & 11 \\ 9-26-74 & 1500 & 0 & 32 & 1.3 & .03 & .50 & .53 & .01 & .01 & 11 \\ 11-14-74 & 1230 & 0 & 26 & 1.1 & .03 & .06 & .09 & .0 & .01 & 25 \\ 1-22-75 & 1330 & 0 & 19 & 1.5 & .04 & .52 & .56 & .01 & .01 & 14 \\ 3-12-75 & 1400 & 0 & 17 & 1.3 & .02 & .42 & .44 & .01 & .01 & 12 \\ 5-14-75 & 0945 & -- & -- & --- & ---- & ---- & ---17 & ---- & ---- & --12 \\ 5-21-75 & 1330 & 0 & 24 & .99 & .01 & .17 & .18 & .0 & .02 & 12\end{array}$


Table 9.--Water-quality determinations in Sacony Creek basin.

[Results in milligrams per litre except as noted]

\begin{tabular}{|c|c|c|c|c|c|c|c|c|c|}
\hline & & & & & & Field & ermin & ons & \\
\hline . & $\begin{array}{l}I \\
0 \\
0\end{array}$ & & & & & 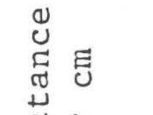 & $\begin{array}{r}\text { Dis } \\
\text { ox } \\
\end{array}$ & $\begin{array}{l}\text { ved } \\
\mathrm{n}\end{array}$ & \\
\hline 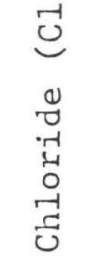 & 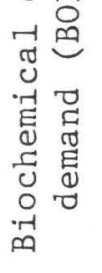 & 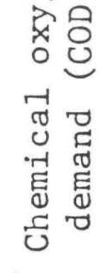 & 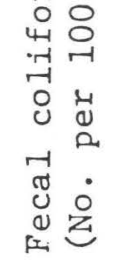 & 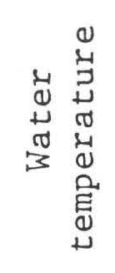 & 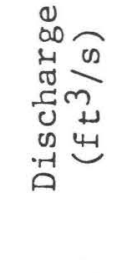 & 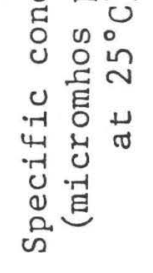 & 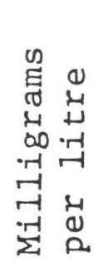 & 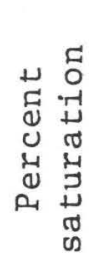 & 垔 \\
\hline & & $1 \mathrm{e}$ & ly $c$ & ne & jers & - (Sta & 1) & & \\
\hline 7.5 & 0.8 & 10 & 630 & 20.0 & 0.55 & 135 & 8.2 & 88 & 7.6 \\
\hline 7.4 & 1.0 & 6.2 & 750 & 12.0 & .92 & 125 & 10.0 & 92 & 7.2 \\
\hline 6.5 & 1.8 & 3 & 18 & 8.5 & 1.10 & 110 & 10.8 & 92 & 7.5 \\
\hline 12 & 1.6 & 0 & 1 & 2.5 & 2.38 & 160 & 13.4 & 97 & 6.9 \\
\hline 7.0 & .9 & 15 & 33 & 3.5 & 7.14 & 110 & 13.7 & 102 & 7.5 \\
\hline---- & --- & ---- & 61 & 14.0 & 4.38 & 105 & 11.0 & 97 & 7.9 \\
\hline 7.0 & .6 & 5.0 & 130 & 16.5 & 3.04 & 105 & 9.6 & 97 & 7.3 \\
\hline
\end{tabular}

Sacony Creek at Sally Ann Furnace, near Bowers, Pa. (Station 2)

\begin{tabular}{rrrrrrrrrr}
2.5 & 0.2 & \multicolumn{1}{c}{29} & 310 & 19.0 & 2.60 & 120 & 8.4 & 90 & 7.7 \\
3.6 & 1.4 & 5.4 & 110 & 13.5 & 1.59 & 120 & 10.2 & 97 & 7.5 \\
4.0 & 1.0 & 9.0 & 88 & 8.0 & 4.24 & 120 & 11.2 & 94 & 7.4 \\
3.0 & 1.0 & 3.0 & 4 & 1.5 & 6.82 & 100 & 14.2 & 100 & 6.8 \\
4.0 & .8 & 7.0 & 18 & 4.0 & 25.4 & 100 & 13.8 & 103 & 7.5 \\
---- & --- & --- & --- & 14.5 & 3.94 & 100 & 11.2 & 108 & 7.8 \\
3.0 & .7 & 6.0 & 160 & 17.0 & 7.81 & 100 & 9.4 & 97 & 7.5
\end{tabular}


Table 9.--Water-quality determinations in Sacony Creek basin--continued.

[Results in milligrams per litre except as noted]

\begin{tabular}{|c|c|c|c|c|c|c|c|c|c|c|}
\hline \multirow[b]{2}{*}{ Date } & \multirow[b]{2}{*}{ Time } & \multicolumn{9}{|c|}{ Laboratory analyses } \\
\hline & & 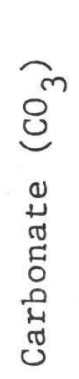 & 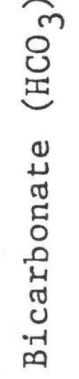 & 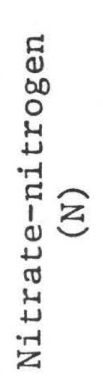 & 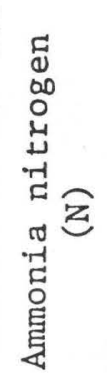 & 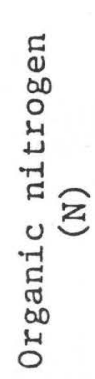 & 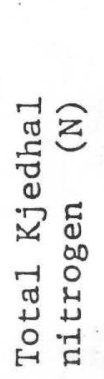 & 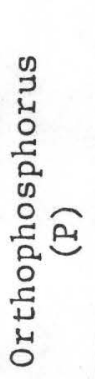 & 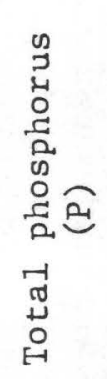 & 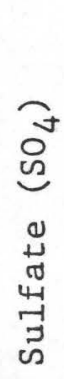 \\
\hline
\end{tabular}

Sacony Creek above Bowers, Pa. (Station 3)

$\begin{array}{rrrrrrrrrrr}7-31-74 & 1130 & 0 & 32 & 1.2 & ---- & ---- & ---- & --- & ---- & 11 \\ 9-26-74 & 1000 & 0 & 34 & 1.0 & .04 & .48 & .52 & .02 & .03 & 12 \\ 11-14-74 & 0950 & 0 & 30 & .99 & .04 & .29 & .33 & .0 & .01 & 18 \\ 1-22-75 & 1045 & 0 & 20 & 1.5 & .04 & .76 & .80 & .01 & .01 & 18 \\ 3-12-75 & 1200 & 0 & 19 & 1.3 & .01 & .29 & .30 & .01 & .01 & 15 \\ 5-14-75 & 0830 & -- & -- & --- & ---- & ---- & ---- & ---- & ---- & -14 \\ 5-21-75 & 1100 & 0 & 27 & .98 & .01 & .03 & .04 & .01 & .02 & 14\end{array}$

Sacony Creek tributary near Bowers, Pa. (Station 4)

$\begin{array}{rrrrrrrrrrr}7-31-74 & 1025 & 0 & 41 & 0.54 & ---- & ---- & ---- & --- & ---- & 15 \\ 9-26-74 & 0820 & 0 & 39 & .56 & .01 & .31 & .32 & .02 & .03 & 15 \\ 11-14-74 & 1040 & 0 & 36 & .61 & .05 & .22 & .27 & .01 & .01 & 22 \\ 1-22-75 & 1000 & 0 & 26 & .93 & .04 & .47 & .51 & .01 & .02 & 22 \\ 3-12-75 & 1000 & 0 & 26 & .90 & .03 & .43 & .46 & .01 & .01 & 19 \\ 5-14-75 & 1400 & -- & -- & --- & ---- & ---- & ---- & ---- & --.- & -19 \\ 5-21-75 & 1245 & 0 & 30 & .56 & .01 & .16 & .17 & .01 & .02 & 19\end{array}$


Table 9.--Water-quality determinations in Sacony Creek basin--continued.

[Results in milligrams per litre except as noted]

\begin{tabular}{|c|c|c|c|c|c|c|c|c|c|}
\hline \multirow[b]{3}{*}{ 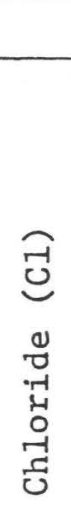 } & \multirow{3}{*}{ 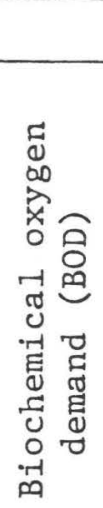 } & \multirow{3}{*}{ 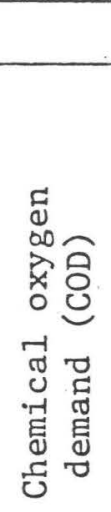 } & \multirow{3}{*}{ 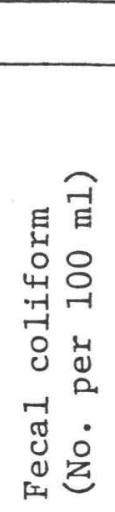 } & \multirow{3}{*}{ 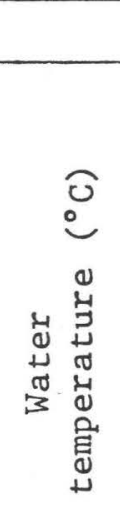 } & \multicolumn{5}{|c|}{ Field determinations } \\
\hline & & & & & \multirow[b]{2}{*}{ 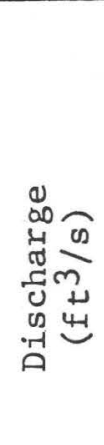 } & \multirow{2}{*}{ 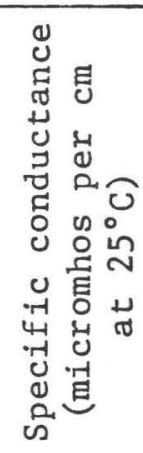 } & \multicolumn{2}{|c|}{$\begin{array}{l}\text { Dissolved } \\
\text { oxygen }\end{array}$} & \\
\hline & & & & & & & 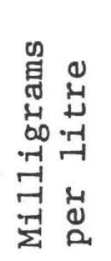 & 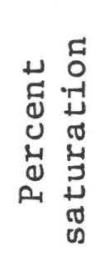 & 窝 \\
\hline
\end{tabular}

Sacony Creek above Bowers, Pa. (Station 3)

$\begin{array}{rrrrrrrrrr}4.0 & 0.2 & 8.0 & 460 & 20.0 & 2.95 & 140 & 8.2 & 90 & 7.5 \\ 4.2 & 1.0 & 6.7 & 530 & 12.0 & 3.89 & 120 & 10.6 & 97 & 7.6 \\ 6.0 & .4 & 9.0 & 100 & 7.5 & 5.37 & 120 & 11.6 & 96 & 7.7 \\ 5.5 & .8 & 4.0 & 3 & .0 & 12.9 & 110 & 14.2 & 98 & 6.8 \\ 5.0 & .6 & 5.0 & 170 & 4.5 & 15.9 & 110 & 13.5 & 103 & 7.6 \\ ---- & -- & -1 .- & 320 & 13.5 & 18.1 & 100 & 11.2 & 106 & 7.9 \\ 5.0 & 1.4 & 9 & 700 & 17.0 & 11.4 & 120 & 10.0 & 102 & 7.2\end{array}$

Sacony Creek tributary near Bowers, Pa. (Station 4)

$\begin{array}{rrrrrrrrrr}2.0 & 0.8 & 10 & 2,600 & 23.0 & 0.58 & 155 & 7.9 & 91 & 7.4 \\ 3.0 & .8 & 4.6 & 3,000 & 12.5 & .42 & 125 & 9.8 & 90 & 6.6 \\ 3.0 & .6 & 8 & 42 & 8.0 & .47 & 130 & 11.2 & 93 & 7.5 \\ 3.0 & 1.0 & 6.0 & 3 & .0 & 1.26 & 120 & 14.0 & 95 & 6.7 \\ 4.0 & .6 & 8.0 & 280 & 4.0 & 2.05 & 120 & 13.6 & 110 & 7.8 \\ ---- & -1 .- & -12 & 530 & 18.5 & 4.19 & 110 & 11.0 & 155 & 7.9 \\ 2.0 & .6 & 12 & 1,200 & 18.0 & 2.48 & 120 & 8.0 & 83 & 7.7\end{array}$


Table 9.--Water-quality determinations in Sacony Creek basin--continued.

[Results in milligrams per litre except as noted]

\begin{tabular}{|c|c|c|c|c|c|c|c|c|c|c|}
\hline \multirow[b]{2}{*}{ Date } & \multirow[b]{2}{*}{ Time } & \multicolumn{9}{|c|}{ Laboratory analyses } \\
\hline & & 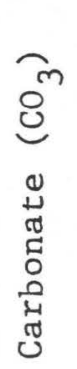 & 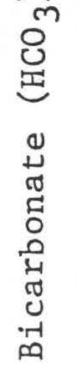 & 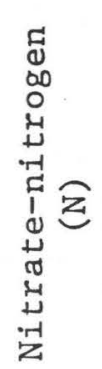 & 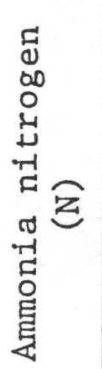 & 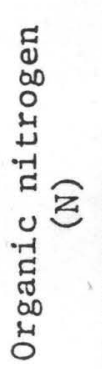 & 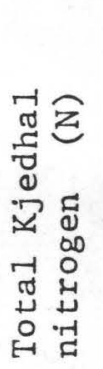 & 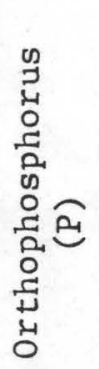 & 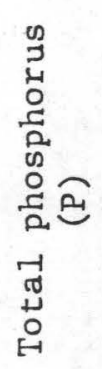 & 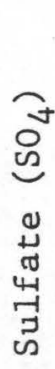 \\
\hline
\end{tabular}

Sacony Creek below Bowers, Pa. (Station 5)

$\begin{array}{rrrrrrrrrrr}7-30-74 & 1845 & 0.0 & 31 & 0.77 & --- & ---- & ---- & --- & ---- & 12 \\ 9-25-74 & 1500 & 1.0 & 31 & .97 & .02 & .48 & .50 & .01 & .02 & 12 \\ 11-13-74 & 1540 & .0 & 27 & .81 & .07 & .32 & .38 & .0 & .01 & 18 \\ 1-22-75 & 0830 & .0 & 21 & 1.6 & .05 & .52 & .57 & .01 & .01 & 17 \\ 3-12-75 & 0845 & .0 & 22 & 1.6 & .01 & .38 & .39 & .01 & .01 & 14 \\ 5-13-75 & 1545 & -- & -- & --- & ---- & ---- & ---- & --- & ---- & --- \\ 5-21-75 & 1500 & 0 & 0 & .93 & .01 & .20 & .21 & .01 & .01 & 0\end{array}$

Sacony Creek above Kutztown, Pa. (Station 6)

$\begin{array}{rrcrrrrrrrr}7-30-74 & 1935 & 0 & 32 & 0.66 & ---- & ---- & ---- & --- & ---- & 13 \\ 9-25-74 & 1315 & .8 & 33 & .99 & .06 & .70 & .76 & .02 & .02 & 11 \\ 11-13-74 & 1450 & 0 & 28 & .81 & .10 & .42 & .52 & .01 & .01 & 19 \\ 1-21-75 & 1430 & 0 & 22 & 1.5 & .05 & .47 & .52 & .01 & .01 & 20 \\ 3-11-75 & 1540 & 0 & 22 & 1.8 & .01 & .43 & .44 & .01 & .01 & 14 \\ 5-13-75 & 1445 & -- & -- & --- & ---- & ---- & ---- & --- & ---- & ---- \\ 5-22-75 & 1130 & 0 & 28 & .93 & .01 & .30 & .31 & .01 & .02 & 15\end{array}$


Table 9.--Water-quality determinations in Sacony Creek basin--continued.

[Results in milligrams per litre except as noted]

\begin{tabular}{|c|c|c|c|c|c|c|c|c|c|}
\hline \multirow[b]{3}{*}{$\begin{array}{l}\widehat{-} \\
0 \\
0 \\
0 \\
-7 \\
0 \\
\overrightarrow{0} \\
\overrightarrow{0}\end{array}$} & \multirow{3}{*}{ 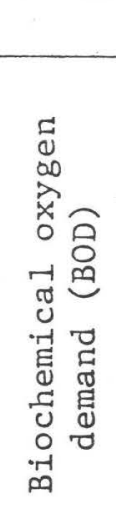 } & \multirow{3}{*}{ 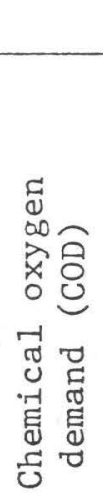 } & \multirow{3}{*}{ 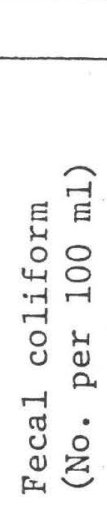 } & \multicolumn{6}{|c|}{ Field determinations } \\
\hline & & & & \multirow{2}{*}{ 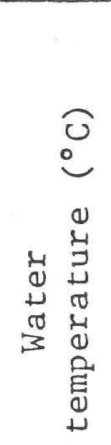 } & \multirow[b]{2}{*}{ 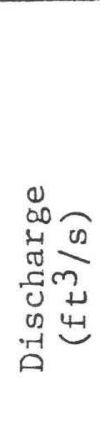 } & \multirow{2}{*}{ 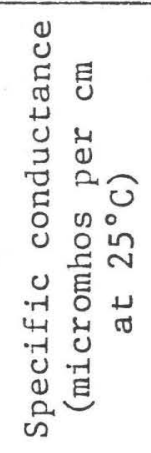 } & \multicolumn{2}{|c|}{$\begin{array}{c}\text { Dissolved } \\
\text { oxygen }\end{array}$} & \\
\hline & & & & & & & 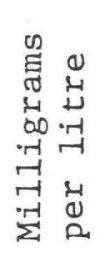 & 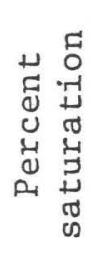 & 蛋 \\
\hline
\end{tabular}

Sacony Creek below Bowers, Pa. (Station 5)

\begin{tabular}{rrrrrrrrrr}
4.0 & 1.2 & 14 & 2,100 & 24.0 & 2.92 & 160 & 7.4 & 86 & 7.4 \\
4.8 & 1.2 & \multicolumn{1}{c}{4.6} & 2,500 & 15.5 & 3.38 & 110 & 10.6 & 105 & 8.5 \\
5.5 & 1.6 & 15 & 900 & 8.5 & 7.73 & 120 & 10.7 & 90 & 7.5 \\
5.5 & 1.4 & 7.2 & 8 & .0 & 12.6 & 100 & 14.2 & 97 & 6.7 \\
5.0 & .6 & 8.0 & 120 & 3.5 & 12.8 & 110 & 13.4 & 100 & 7.7 \\
--- & --- & --- & 410 & 19.5 & 14.1 & 105 & 10.0 & 108 & 8.4 \\
4.5 & 1.0 & 12 & 120 & 19.5 & 11.8 & 95 & 9.6 & 103 & 8.3
\end{tabular}

Sacony Creek above Kutztown, Pa. (Station 6)

$\begin{array}{rrrrrrrrrr}3.5 & 2.2 & 18 & 12,000 & 24.0 & 3.97 & 130 & 6.8 & 78 & 7.7 \\ 4.6 & 1.6 & 2.9 & 9,600 & 16.5 & 3.72 & 130 & 11.9 & 120 & 8.4 \\ 6.5 & 1.6 & 19 & 1,300 & 9.0 & 9.31 & 130 & 10.5 & 90 & 7.6 \\ 6.0 & 1.0 & 8.0 & 1 & 4.5 & 17.1 & 120 & 14.2 & 108 & 7.4 \\ 5.0 & .4 & 7.0 & 120 & 6.5 & 13.7 & 120 & 13.4 & 107 & 8.1 \\ --- & -- & ---- & 700 & 20.5 & 16.1 & 120 & 10.2 & 111 & 8.6 \\ 4.0 & 1.0 & 10 & 2,500 & 21.0 & 12.3 & 115 & 10.0 & 110 & 8.4\end{array}$


Table 9.--Water-quality determinations in Sacony Creek basin--continued.

[Results in milligrams per litre except as noted]

\begin{tabular}{|c|c|c|c|c|c|c|c|c|c|c|}
\hline \multirow[b]{2}{*}{ Date } & \multirow[b]{2}{*}{ Time } & \multicolumn{9}{|c|}{ Laboratory analyses } \\
\hline & & 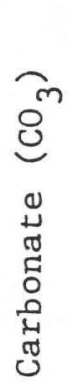 & 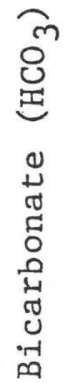 & 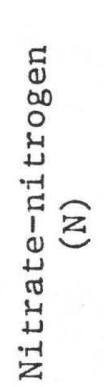 & 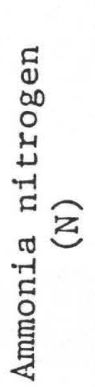 & 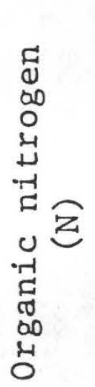 & 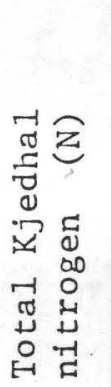 & 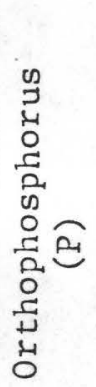 & 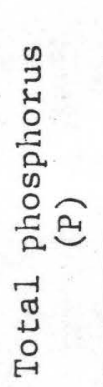 & 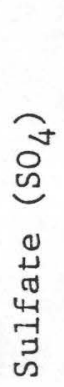 \\
\hline
\end{tabular}

Sacony Creek at Normal Avenue, at Kutztown, Pa. (Station 7)

$\begin{array}{rrrrrrrrrrr}7-30-74 & 0930 & 0 & 84 & 2.7 & ---- & ---- & ---- & --- & ---- & 19 \\ 9-25-74 & 1100 & 0 & 167 & 5.0 & .02 & .46 & .48 & .03 & .04 & 27 \\ 11-13-74 & 1345 & 0 & 76 & 1.8 & .14 & .86 & 1.0 & .01 & .02 & 25 \\ 1-21-75 & 1340 & 0 & 128 & 4.2 & .05 & .99 & 1.04 & .03 & .03 & 30 \\ 3-11-75 & 1445 & 3.0 & 145 & 5.0 & .01 & .59 & .60 & .02 & .02 & 28 \\ 5-13-75 & 1345 & -- & -- & --- & ---- & ---- & ---- & --- & ---- & ---- \\ 5-22-75 & 1020 & 0 & 138 & 9.7 & .01 & .04 & .05 & .01 & .02 & 26\end{array}$

Sacony Creek at Kutztown, Pa. (Station 8)

$\begin{array}{rrrrrrrrrrr}7-30-74 & 1200 & 0 & 95 & 2.5 & ---- & ---- & ---- & --- & ---- & 20 \\ 9-25-74 & 0800 & 0 & 187 & 4.5 & .03 & .56 & .59 & .02 & .02 & 28 \\ 11-13-74 & 1315 & 0 & 84 & 1.6 & .11 & .75 & .86 & .02 & .02 & 26 \\ 1-21-75 & 1245 & 0 & 169 & 4.3 & .05 & .80 & .85 & .02 & .03 & 37 \\ 3-11-75 & 1330 & 3.0 & 151 & .50 & .06 & .42 & .48 & .02 & .02 & 29 \\ 5-13-75 & 1300 & -- & -- & --- & ---- & ---- & ---- & --- & ---- & ---1 \\ 5-22-75 & 0920 & 0 & 149 & 3.8 & .01 & .12 & .13 & .02 & .03 & 25\end{array}$


Table 9 --Water-quality determinations in Sacony Creekbasin--continued.

[Results in milligrams per litre except as noted]

\begin{tabular}{|c|c|c|c|c|c|c|c|c|c|}
\hline \multirow[b]{3}{*}{ 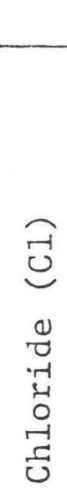 } & & & & & & Field & ermin & ons & \\
\hline & \multirow{2}{*}{ 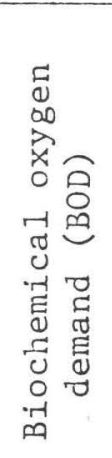 } & \multirow{2}{*}{ 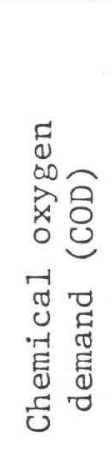 } & \multirow{2}{*}{ 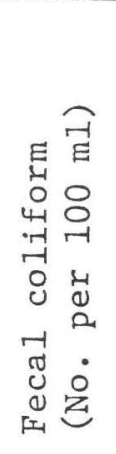 } & \multirow{2}{*}{ 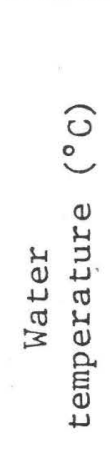 } & \multirow[b]{2}{*}{ 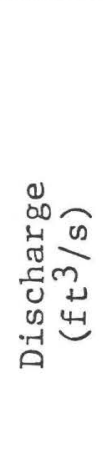 } & \multirow{2}{*}{ 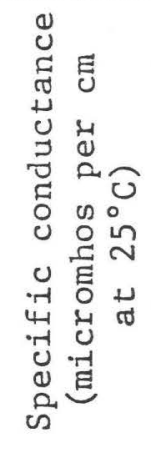 } & \multicolumn{2}{|c|}{$\begin{array}{c}\text { Dissolved } \\
\text { oxygen }\end{array}$} & \\
\hline & & & & & & & 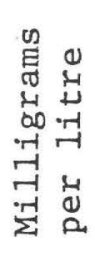 & 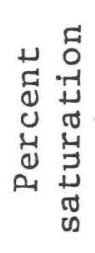 & 贾 \\
\hline
\end{tabular}

Sacony Creek at Normal Avenue, at Kutztown, Pa. (Station 7)

\begin{tabular}{rrrrrrrrrr}
6.0 & 2.2 & \multicolumn{1}{c}{21} & 19,000 & 19.0 & 15.8 & 255 & 6.9 & 73 & 7.3 \\
30 & 1.8 & \multicolumn{1}{c}{4.2} & 16,000 & 12.0 & 8.11 & 475 & 10.2 & 93 & 7.4 \\
9.0 & 2.2 & 22 & 4,000 & 9.0 & 14.2 & 250 & 10.0 & 85 & 7.4 \\
9.5 & 2.4 & 7.0 & 75 & 4.0 & 24.8 & 370 & 12.6 & 95 & 7.3 \\
8.5 & .1 & 10.0 & 69 & 8.0 & 34.5 & 390 & 13.4 & 111 & 7.9 \\
---- & -- & --- & 600 & 17.5 & 33.3 & 320 & 11.8 & 120 & 8.0 \\
8.0 & .6 & 8.0 & 1,600 & 16.0 & 23.6 & 340 & 9.8 & 97 & 7.7
\end{tabular}

Sacony Creek at Kutztown, Pa. ( Śtation 8)

\begin{tabular}{rrrrrrrrrr}
7.0 & 2.8 & \multicolumn{1}{c}{16} & 7,600 & 22.0 & 13.8 & 270 & 8.5 & 95 & 7.8 \\
60 & 1.6 & \multicolumn{1}{c}{8.3} & 4,500 & 10.5 & 10.3 & 520 & 11.0 & 97 & 7.4 \\
9.0 & 2.4 & 19 & 9,000 & 9.5 & 17.3 & 260 & 10.4 & 90 & 7.4 \\
12 & 1.4 & 7.0 & 67 & 3.0 & 22.6 & 480 & 13.7 & 100 & 7.3 \\
10 & .2 & 7.0 & 46 & 8.5 & 35.3 & 410 & 13.8 & 116 & 8.1 \\
--- & -- & --- & 640 & 16.5 & 36.3 & 340 & 12.2 & 123 & 8.2 \\
9.0 & .6 & 6.0 & 1,800 & 15.5 & 25.3 & 360 & 10.4 & 103 & 7.9
\end{tabular}


Table 9.--Water-quality determinations in Sacony Creek basin--continued.

[Results in milligrams per litre except as noted]

\begin{tabular}{|c|c|c|c|c|c|c|c|c|c|c|}
\hline & & \multicolumn{9}{|c|}{ Laboratory analyses } \\
\hline Date & Time & 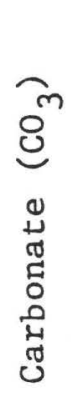 & 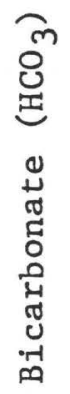 & 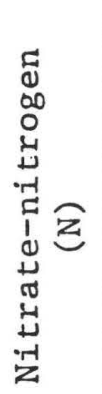 & 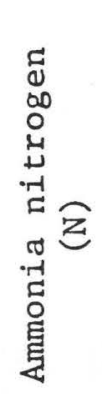 & 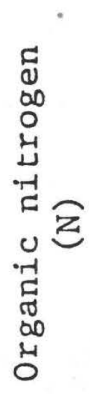 & 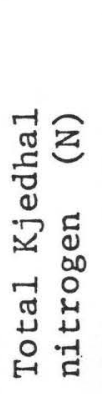 & 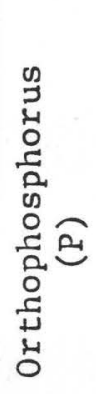 & 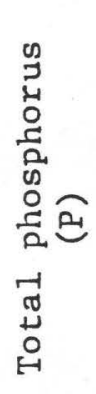 & 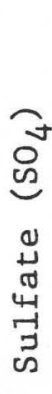 \\
\hline
\end{tabular}

Sacony Creek below Kutztown, Pa. (Station 9)

\begin{tabular}{|c|c|c|c|c|c|c|c|c|c|c|}
\hline $7-30-74$ & 1330 & 0.0 & 106 & 3.2 & --- & --- & --- & --- & --- & 24 \\
\hline $9-24-74$ & 1600 & .0 & 194 & 5.0 & 1.04 & 1.28 & 2.32 & .47 & .54 & 38 \\
\hline $11-13-74$ & 1140 & .0 & 96 & 2.3 & .50 & .93 & 1.4 & .27 & . 31 & 34 \\
\hline $1-21-75$ & 1120 & .0 & 170 & 4.9 & .29 & .77 & 1.06 & .13 & .15 & 49 \\
\hline $3-11-75$ & 1145 & 1.0 & 164 & 5.2 & .59 & 1.1 & 1.69 & .22 & .23 & 33 \\
\hline $5-13-75$ & 1150 & -- & -- & --- & ---- & ---- & ---- & ---- & ---- & -- \\
\hline $5-22-75$ & 0845 & .0 & 156 & 4.1 & .01 & .27 & .28 & .22 & .25 & 28 \\
\hline
\end{tabular}

Sacony Creek at Greenwich Bridge, near Kutztown, Pa. (Station 10)

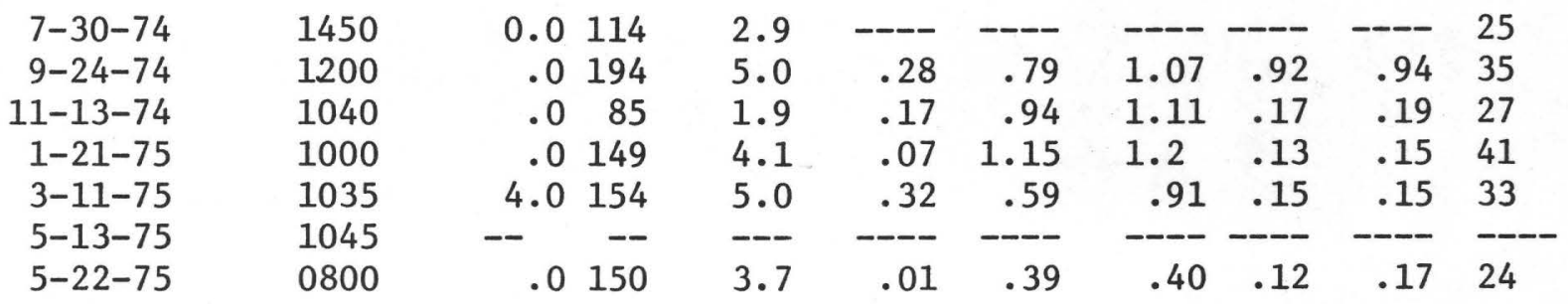


Table 9.--Water-quality determinations in Sacony Creek basin--continued.

[Results in milligrams per litre except as noted]

\begin{tabular}{|c|c|c|c|c|c|c|c|c|c|}
\hline \multirow[b]{3}{*}{ 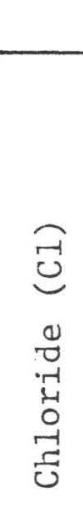 } & \multirow{3}{*}{ 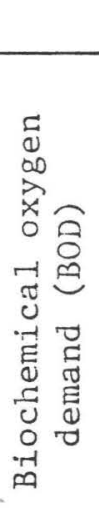 } & \multirow[b]{3}{*}{ 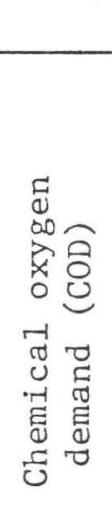 } & \multirow{3}{*}{ 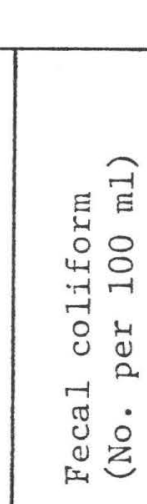 } & \multicolumn{6}{|c|}{ Field determinations } \\
\hline & & & & & \multirow[b]{2}{*}{ 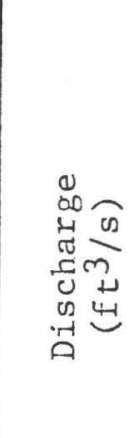 } & \multirow{2}{*}{ 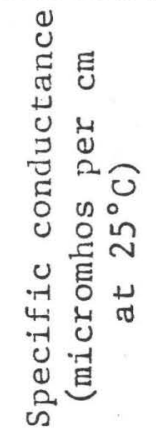 } & \multicolumn{2}{|c|}{$\begin{array}{c}\text { Dissolved } \\
\text { oxygen }\end{array}$} & \multirow[b]{2}{*}{ 贾 } \\
\hline & & & & 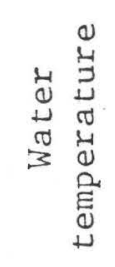 & & & \multirow[t]{2}{*}{ 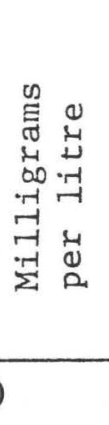 } & \multirow[t]{2}{*}{ 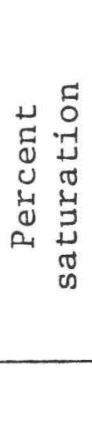 } & \\
\hline \multicolumn{8}{|c|}{ Sacony Creek below Kutztown, Pa. (Station 9) } & & \\
\hline 20 & 3.4 & 20 & 6,200 & 24.0 & 14.9 & 340 & 8.8 & 103 & 7.8 \\
\hline 48 & 4.8 & 13 & 11,000 & 14.5 & 12.2 & 680 & 13.2 & 127 & 8.0 \\
\hline 21 & 3.6 & 19.0 & 6,300 & 9.0 & 23.3 & 340 & 10.0 & 85 & 7.3 \\
\hline 20 & 2.6 & 7.0 & 1,400 & 1.5 & 23.6 & 520 & 13.8 & 98 & 7.3 \\
\hline 22 & .2 & 6.0 & 100 & 7.0 & 38.2 & 490 & 13.5 & 110 & 8.0 \\
\hline--- & --- & ---- & 2,300 & 15.5 & 46.1 & 380 & 11.4 & 113 & 7.8 \\
\hline 18 & 1.2 & 9.0 & 2,400 & 15.5 & 29.9 & 395 & 9.6 & 95 & 7.8 \\
\hline
\end{tabular}

Sacony Creek at Greenwich Bridge, near Kutztown, Pa. (Station 10)

$\begin{array}{rrrrrrrrrr}19 & 2.6 & 21 & 3,200 & 24.0 & 17.0 & 350 & 7.4 & 87 & 7.8 \\ 27 & 2.1 & 11.0 & 4,700 & 11.0 & 12.8 & 530 & 9.3 & 83 & 7.5 \\ 14 & 1.2 & 14 & 2,500 & 9.0 & 28.9 & 280 & 9.6 & 82 & 7.3 \\ 27 & 1.8 & 6.0 & 1,300 & .5 & 24.1 & 480 & 13.8 & 95 & 7.4 \\ 24 & .7 & 5.0 & 280 & 6.5 & 41.0 & 490 & 12.4 & 100 & 7.6 \\ ---- & -- & --- & 1,100 & 15.0 & 44.0 & 350 & 11.0 & 107 & 7.8 \\ 14 & 1.2 & 1.2 & 700 & 16.0 & 27.5 & 370 & 8.4 & 84 & 7.5\end{array}$

